\title{
SINGLY GENERATED QUASIVARIETIES AND RESIDUATED STRUCTURES
}

\author{
T. MORASCHINI, J.G. RAFTERY, AND J.J. WANNENBURG
}

\begin{abstract}
A quasivariety $\mathrm{K}$ of algebras has the joint embedding property (JEP) iff it is generated by a single algebra $\boldsymbol{A}$. It is structurally complete iff the free $\aleph_{0}$-generated algebra in $\mathrm{K}$ can serve as $\boldsymbol{A}$. A consequence of this demand, called 'passive structural completeness' (PSC), is that the nontrivial members of $\mathrm{K}$ all satisfy the same existential positive sentences. We prove that if $\mathrm{K}$ is PSC then it still has the JEP, and if it has the JEP and its nontrivial members lack trivial subalgebras, then its relatively simple members all belong to the universal class generated by one of them. Under these conditions, if $\mathrm{K}$ is relatively semisimple then it is generated by one $\mathrm{K}$-simple algebra. It is a minimal quasivariety if, moreover, it is PSC but fails to unify some finite set of equations. We also prove that a quasivariety of finite type, with a finite nontrivial member, is PSC iff its nontrivial members have a common retract. The theory is then applied to the variety of De Morgan monoids, where we isolate the sub(quasi)varieties that are PSC and those that have the JEP, while throwing fresh light on those that are structurally complete. The results illuminate the extension lattices of intuitionistic and relevance logics.
\end{abstract}

\section{INTRODUCTION}

Familiar logics often have an algebraic counterpart that is a quasivariety $\mathrm{K}$ of algebras; in many cases it is a variety. In this situation, the derivable inference rules of the logic may or may not be determined by a single set of 'truth tables', i.e., by the operation tables of a single algebra $\boldsymbol{A} \in \mathrm{K}$. If some member of $\mathrm{K}$ determines the finite rules of the logic, then another member determines all of the rules (see Remark [5.13), so what is needed is

Key words and phrases. Joint embedding property, (passive) structural completeness, retract, De Morgan monoid, Dunn monoid, Brouwerian algebra.

2010 Mathematics Subject Classification.

Primary: 03B47, 06F05, 08C15. Secondary: 03B55, 03G25, 06D20.

This work received funding from the European Union's Horizon 2020 research and innovation programme under the Marie Sklodowska-Curie grant agreement No 689176 (project "Syntax Meets Semantics: Methods, Interactions, and Connections in Substructural logics"). The first author was supported by project CZ.02.2.69/0.0/0.0/17_050/0008361, OPVVV MŠMT, MSCA-IF Lidské zdroje v teoretické informatice, and by RVO 67985807. The second author was supported in part by the National Research Foundation of South Africa (UID 85407). The third author was supported by the DST-NRF Centre of Excellence in Mathematical and Statistical Sciences (CoE-MaSS), South Africa. The University of Pretoria Staff Exchange Bursary Programme and the CoE-MaSS (RT18ALG/006) partially funded the first author's travel to Pretoria in 2017 and 2018, respectively. Opinions expressed and conclusions arrived at are those of the authors and are not necessarily to be attributed to the CoE-MaSS. 
only that $\mathrm{K}$ be generated by a single algebra. Even when $\mathrm{K}$ is a variety, it must be generated as a quasivariety by one of its members, if the generator is to determine rules (as opposed to theorems only).

Obviously, classical propositional logic has this property: its algebraic counterpart - the variety of Boolean algebras - is generated as a quasivariety by its unique two-element member. More surprisingly, the same holds for intuitionistic propositional logic (though not with a finite algebra), and for the relevance logic $\mathbf{R}$ [65], but not for its conservative expansion $\mathbf{R}^{\mathbf{t}}$ (with the so-called Ackermann constants of [1]). In the intuitionistic case, the algebra determining the (possibly infinite) rules cannot be countable [66].

Maltsev [40] proved that a quasivariety $\mathrm{K}$ is generated by a single algebra iff it has the joint embedding property (JEP), i.e., any two nontrivial members of $\mathrm{K}$ can both be embedded into some third member. Los and Suszko [36] characterized this demand by a syntactic 'relevance principle' (finitized in Definition 5.11 below). Various strengthenings of the JEP have received attention in the literature. Their names reflect logical origins, but we choose maximally transparent characterizations here as definitions. One such strengthening, called structural completeness, asks (in effect) that a quasivariety be generated by its free $\aleph_{0}$-generated member. A weaker variant, now called passive structural completeness (PSC), amounts to the demand that any two nontrivial members of $\mathrm{K}$ have the same existential positive theory. This hereditary property still implies the JEP (Theorem 4.3).

Our original goal was to investigate these properties for classes of De Morgan monoids (i.e., the models of $\mathbf{R}^{\mathbf{t}}$ ). It became clear, however, that in many of our results, large parts of the proofs had a general universal algebraic (or even model-theoretic) character, so the first half of this paper concerns such generalities. We call $\mathrm{K}$ a Kollár quasivariety (after [33]) if its nontrivial members lack trivial subalgebras. We prove that if such a quasivariety has the JEP, then its relatively simple members all belong to the universal class generated by one of them (Theorem [5.6). If, in addition, $\mathrm{K}$ is relatively semisimple, then it is generated (as a quasivariety) by one $\mathrm{K}$-simple algebra. We prove that a quasivariety of finite type with a finite nontrivial member is PSC iff its nontrivial members have a common retract (Theorem [7.6).

The second half of the paper deals with (quasi)varieties of De Morgan monoids, and some closely related residuated structures. Among other results, we describe completely the varieties of De Morgan monoids that are PSC (Theorem 8.7), and characterize those with the JEP (Theorem 8.8). The structurally complete varieties of De Morgan monoids fall into two classes - a denumerable family that is fully transparent and a more opaque collection of subvarieties of a certain PSC variety $M$. We prove that this $M$ also has uncountably many structurally incomplete subvarieties, by exhibiting $2^{\aleph_{0}}$ structurally incomplete varieties of Brouwerian algebras (of depth 3) and applying a 'reflection' construction (Theorems 9.6 and 10.8). 


\section{Preliminaries}

We deal with structures $\boldsymbol{A}=\langle A ; F ; R\rangle$, where $F$ [resp. $R$ ] is a set of finitary operations [resp. relations] on the non-empty set $A$. Constants, i.e., distinguished elements of $\boldsymbol{A}$, are treated as nullary basic operations. We call $\boldsymbol{A}$ finite [resp. trivial] if its universe $A$ is finite [resp. $|A|=1$ and $R$ consists of non-empty relations]. Of course, structures model first-order signatures, a.k.a. types. An infinite set $\operatorname{Var}$ of variables is fixed for the entire discussion. Formulas of any kind are assumed to involve only variables from Var. Recall that a first-order formula with no free variable is called a sentence. For a set $\Sigma$ of first-order formulas, the notation $\mathrm{K} \models \Sigma$ means that the universal closure $\forall \bar{x} \Phi$ of each $\Phi \in \Sigma$ is true in every structure belonging to $\mathrm{K}$.

An atomic formula of a first-order signature is a formal equation $\alpha \approx \beta$ between terms or an expression $r\left(\alpha_{1}, \ldots, \alpha_{m}\right)$, where $r$ is a basic relation symbol and each $\alpha_{j}$ a term. A strict universal Horn formula has the form

$$
\left(\&_{i<n} \Phi_{i}\right) \Longrightarrow \Phi_{n}
$$

where $n \in \omega$ and $\Phi_{0}, \ldots, \Phi_{n}$ are atomic formulas. We call (11) a quasiequation if $\Phi_{0}, \ldots, \Phi_{n}$ are equations. A variety [resp. quasivariety] is the model class of a set of atomic [resp. strict universal Horn] formulas. It is said to be algebraic if it consists of algebras $\boldsymbol{A}=\langle A ; F\rangle$, i.e., of structures with no indicated relation.

The class operator symbols $\mathbb{I}, \mathbb{H}, \mathbb{S}, \mathbb{E}, \mathbb{P}, \mathbb{P}_{\mathrm{S}}, \mathbb{P}_{\mathrm{U}}$ and $\mathbb{R}_{\mathrm{U}}$ stand for closure under isomorphic and homomorphic (surjective) images, substructures, extensions (i.e., superstructures), direct and subdirect products, ultraproducts and ultraroots, respectively. Homomorphisms between similar structures are assumed to preserve basic relations (as well as operations), but they need not reflect the relations. An isomorphism is a bijective homomorphism whose inverse function is also a homomorphism. An embedding of a structure $\boldsymbol{A}$ into a structure $\boldsymbol{B}$ is an isomorphism from $\boldsymbol{A}$ onto a substructure of $\boldsymbol{B}$. In a given signature, the direct product of the empty family is interpreted as a trivial structure. For each class operator $\mathbb{O}$, we abbreviate $\mathbb{O}\left(\left\{\boldsymbol{A}_{1}, \ldots, \boldsymbol{A}_{n}\right\}\right)$ as $\mathbb{O}\left(\boldsymbol{A}_{1}, \ldots, \boldsymbol{A}_{n}\right)$.

Let $\mathrm{K}$ be a class of similar structures. We say that $\mathrm{K}$ is elementary [resp. universal] if it is the model class of a set of first-order [resp. universal firstorder] sentences. This amounts to the demand that $\mathrm{K}$ be closed under $\mathbb{R}_{U}$ [resp. $\left.\mathbb{S}\right], \mathbb{I}$ and $\mathbb{P}_{U}$. Two similar structures are elementarily equivalent if they satisfy the same first-order sentences. The smallest variety [resp. quasivariety] containing $\mathrm{K}$ is $\mathbb{V}(\mathrm{K}):=\mathbb{H} \mathbb{S P}(\mathrm{K})\left[\operatorname{resp} . \mathbb{Q}(\mathrm{K}):=\mathbb{I S P P}_{\mathrm{U}}(\mathrm{K})\right]$. The origins of these claims are discussed in [25, Ch. 2], where proofs can also be found. Recall that $\mathbb{P}_{\mathrm{U}}(\mathrm{K}) \subseteq \mathbb{I}(\mathrm{K})$ if $\mathrm{K}$ is a finite set of finite structures.

Let $\mathrm{K}$ be a quasivariety, with $\boldsymbol{A} \in \mathrm{K}$. We say that $\boldsymbol{A}$ is [finitely] Ksubdirectly irreducible if the following is true for every [finite non-empty] set $I$ and every family $\left\{\boldsymbol{A}_{i}: i \in I\right\}$ of members of K: whenever an embedding 
$h: \boldsymbol{A} \rightarrow \prod_{I} \boldsymbol{A}_{i}$ is subdirect (i.e., $\pi_{j} h[A]=A_{j}$ for each of the projections $\pi_{j}: \prod_{I} \boldsymbol{A}_{i} \rightarrow \boldsymbol{A}_{j}$ ), then $\pi_{i} \circ h: \boldsymbol{A} \cong \boldsymbol{A}_{i}$ for some $i \in I$. We say that $\boldsymbol{A}$ is $\mathrm{K}$ simple if it is nontrivial and every homomorphism from $\boldsymbol{A}$ onto a nontrivial member of $\mathrm{K}$ is an isomorphism. The prefix ' $\mathrm{K}$-' is often replaced by the word 'relatively' when $\mathrm{K}$ is understood; it is redundant when $\mathrm{K}$ is a variety. We denote by $\mathrm{K}_{R S I}$ [resp. $\mathrm{K}_{R F S I} ; \mathrm{K}_{R S}$ ] the class of $\mathrm{K}$-subdirectly irreducible [resp. finitely $\mathrm{K}$-subdirectly irreducible; K-simple] members of $\mathrm{K}$. Thus, $\mathrm{K}_{R S} \subseteq \mathrm{K}_{R S I} \subseteq \mathrm{K}_{R F S I}$, and $\mathrm{K}_{R S I}$ includes no trivial structure. The analogue of Birkhoff's subdirect decomposition theorem holds, i.e., $\mathrm{K}=\mathbb{I P}_{S}\left(\mathrm{~K}_{R S I}\right)[25$, Thm. 3.1.1]. If every K-subdirectly irreducible member of $\mathrm{K}$ is $\mathrm{K}$-simple, then $\mathrm{K}$ is said to be relatively semisimple.

Because the image of a homomorphism is always a substructure of the co-domain, these definitions have the following consequence.

Fact 2.1. If $h: \boldsymbol{A} \rightarrow \boldsymbol{B}$ is a homomorphism between members of a quasivariety, where $\boldsymbol{A}$ is relatively simple and $\boldsymbol{B}$ has no trivial substructure, then $h$ is an embedding.

\section{Existential Positive Sentences}

Recall that, up to logical equivalence, an existential positive sentence is a first-order sentence of the form $\exists x_{1} \ldots \exists x_{n} \Phi$, where $\Phi$ is a (quantifierfree) disjunction of conjunctions of atomic formulas. Such sentences have a central place in the model theory of 'positive logic' (see [54], for instance). For present purposes, their main significance derives from Theorems 3.1 and 4.3 below. They may be variable-free (and hence quantifier-free).

Given a structure $\boldsymbol{A}=\langle A ; F ; R\rangle$, with $S \subseteq A$, let $\boldsymbol{A}_{S}=\left\langle A ; F \cup S_{0} ; R\right\rangle$, where $S_{0}$ consists of the elements of $S$, treated as new nullary operations on $A$. Let $\operatorname{Th}(\boldsymbol{A})[\operatorname{resp} . \operatorname{Diag}(\boldsymbol{A})]$ denote the set of all [resp. all atomic] firstorder sentences that are true in $\boldsymbol{A}_{A}$. A substructure $\boldsymbol{B}$ of $\boldsymbol{A}$ is called an elementary substructure (and $\boldsymbol{A}$ an elementary extension of $\boldsymbol{B}$ ) if $\boldsymbol{A}_{B} \models \operatorname{Th}(\boldsymbol{B})$. In this case $\boldsymbol{A}$ and $\boldsymbol{B}$ are elementarily equivalent. An embedding is elementary if its image is an elementary substructure of its co-domain. Every structure is elementarily embeddable into each of its ultrapowers.

Theorem 3.1. Let $\boldsymbol{A}$ and $\boldsymbol{B}$ be similar structures. Then $\boldsymbol{B}$ satisfies every existential positive sentence that is true in $\boldsymbol{A}$ iff there is a homomorphism from $\boldsymbol{A}$ into an ultrapower of $\boldsymbol{B}$.

Proof. $(\Rightarrow)$ Let $\Sigma$ be a finite subset of $\operatorname{Diag}(\boldsymbol{A})$. By assumption, $\Sigma \cup \operatorname{Th}(\boldsymbol{B})$ has a model that is an expansion of $\boldsymbol{B}_{B}$ by suitable interpretations in $B$ of the elements of $A$ occurring (as constant symbols) in $\Sigma$. By the Compactness Theorem, therefore, $\operatorname{Diag}(\boldsymbol{A}) \cup \operatorname{Th}(\boldsymbol{B})$ has a model, $\boldsymbol{C}$, say. Let $\boldsymbol{C}^{-}$be the reduct of $\boldsymbol{C}$ in the signature of $\boldsymbol{A}, \boldsymbol{B}$. Now $\boldsymbol{C}^{-}$is isomorphic to an elementary extension of $\boldsymbol{B}$, because $\boldsymbol{C}$ is a model of $\operatorname{Th}(\boldsymbol{B})$. (In particular, the negated atomic sentences in $\operatorname{Th}(\boldsymbol{B})$ separate the elements of $B$.) 
Also, there is a homomorphism from $\boldsymbol{A}$ into $\boldsymbol{C}^{-}$, because $\boldsymbol{C}$ is a model of $\operatorname{Diag}(\boldsymbol{A})$. As $\boldsymbol{C}^{-}$and $\boldsymbol{B}$ are elementarily equivalent, some ultrapower $\boldsymbol{U}$ of $\boldsymbol{C}^{-}$is isomorphic to an ultrapower of $\boldsymbol{B}$, by the Keisler-Shelah Isomorphism Theorem [10, Thm.6.1.15]. Since $\boldsymbol{C}^{-}$is (elementarily) embeddable into $\boldsymbol{U}$, there is a homomorphism from $\boldsymbol{A}$ into $\boldsymbol{U}$.

$(\Leftarrow)$ Clearly, existential positive sentences persist in homomorphic images, in extensions and in ultraroots.

Corollary 3.2. The model class of the set of existential positive sentences satisfied by a structure $\boldsymbol{A}$ is $\mathbb{R}_{\mathrm{U}} \mathbb{E} \mathbb{H}(\boldsymbol{A})$.

Corollary 3.3. The following demands on a quasivariety $\mathrm{K}$ are equivalent.

(i) The nontrivial members of $\mathrm{K}$ all satisfy the same existential positive sentences.

(ii) For any two nontrivial members of $\mathrm{K}$, each can be mapped homomorphically into an ultrapower of the other.

Definition 3.4. A quasivariety is said to be passively structurally complete (PSC) if it satisfies the equivalent conditions of Corollary 3.3. (The terminology will be justified in Sections 6 and 7.)

\section{The Joint Embedding Property}

Definition 4.1. A class $\mathrm{K}$ of similar structures is said to have the joint embedding property (JEP) if, for any two nontrivial structures $\boldsymbol{A}, \boldsymbol{B} \in \mathrm{K}$, there exists $\boldsymbol{C} \in \mathrm{K}$ such that $\boldsymbol{A}$ and $\boldsymbol{B}$ can both be embedded into $\boldsymbol{C}$.

For quasivarieties, the characterization of the JEP given below was proved in [40, Thm. 4] (also see [42, p. 288] or [25, Prop. 2.1.19]).

Theorem 4.2. (Maltsev) A quasivariety $\mathrm{K}$ has the JEP iff it is generated by a single structure (i.e., there exists $\boldsymbol{A} \in \mathrm{K}$ such that $\mathrm{K}=\mathbb{Q}(\boldsymbol{A})$ ).

The following additional characterizations of the JEP for a quasivariety $\mathrm{K}$ are known 1

(i) For each set $\mathrm{S}$ of nontrivial members of $\mathrm{K}$, there exists a member of $\mathrm{K}$ into which every member of $\mathrm{S}$ embeds.

(ii) Whenever $\Phi$ and $\Psi$ are universal sentences whose disjunction $\Phi \sqcup \Psi$ is true in all nontrivial members of $\mathrm{K}$, then there exists $\Xi \in\{\Phi, \Psi\}$ such that $\Xi$ is true in every nontrivial member of $\mathrm{K}$.

(iii) Whenever $\Phi$ and $\Psi$ are existential sentences, each of which is true in some nontrivial member of $\mathrm{K}$, then their conjunction $\Phi \& \Psi$ is true in some nontrivial member of $\mathrm{K}$.

\footnotetext{
${ }^{1}$ They are specializations of conditions in [28, Thm. 1.2], where the JEP was formulated for arbitrary first-order theories, without the restriction to nontrivial models. It is pointed out in 28] that their equivalence with the JEP follows from a result proved in 69.
} 
(iv) Whenever $\Sigma$ is a set of existential sentences, each of which is true in at least one nontrivial member of $\mathrm{K}$, then there is a nontrivial member of $\mathrm{K}$ in which all sentences from $\Sigma$ are true.

Easily, (ii) and (iii) follow from the JEP, and (iv) from (ii). To prove (ii), we apply the Compactness Theorem to $\Sigma \cup\{\overline{\operatorname{Diag}}(\boldsymbol{A}): \boldsymbol{A} \in \mathrm{S}\}$, where $\Sigma$ is a set of sentences axiomatizing $\mathrm{K}$ and each $\overline{\operatorname{Diag}}(\boldsymbol{A})$ is the set of atomic or negated atomic sentences that are true in $\boldsymbol{A}_{A}$. (We arrange first that the members of $\mathrm{S}$ are disjoint.)

Because the JEP need not persist in sub(quasi)varieties, the following result is of interest.

Theorem 4.3. If a quasivariety is PSC (see Definition 3.4), then it has the JEP, and so do all of its subquasivarieties.

Proof. Let $\boldsymbol{A}, \boldsymbol{B}$ be nontrivial members of a PSC quasivariety $\mathrm{K}$. Then there are homomorphisms $f: \boldsymbol{A} \rightarrow \boldsymbol{B}_{u}$ and $g: \boldsymbol{B} \rightarrow \boldsymbol{A}_{u}$, for suitable ultrapowers $\boldsymbol{A}_{u}$ and $\boldsymbol{B}_{u}$ of $\boldsymbol{A}$ and $\boldsymbol{B}$, respectively. Recall that there are (elementary) embeddings $e_{A}: \boldsymbol{A} \rightarrow \boldsymbol{A}_{u}$ and $e_{B}: \boldsymbol{B} \rightarrow \boldsymbol{B}_{u}$. Consider the maps

$$
\left\langle e_{A}, f\right\rangle: \boldsymbol{A} \rightarrow \boldsymbol{A}_{u} \times \boldsymbol{B}_{u} \text { and }\left\langle g, e_{B}\right\rangle: \boldsymbol{B} \rightarrow \boldsymbol{A}_{u} \times \boldsymbol{B}_{u}
$$

defined by the following rules: for every $a \in A$ and $b \in B$,

$$
\left\langle e_{A}, f\right\rangle(a)=\left\langle e_{A}(a), f(a)\right\rangle \text { and }\left\langle g, e_{B}\right\rangle(b)=\left\langle g(b), e_{B}(b)\right\rangle .
$$

Clearly, $\left\langle e_{A}, f\right\rangle$ and $\left\langle g, e_{B}\right\rangle$ are embeddings, so $\boldsymbol{A}, \boldsymbol{B} \in \mathbb{I} S\left(\boldsymbol{A}_{u} \times \boldsymbol{B}_{u}\right)$, and $\boldsymbol{A}_{u} \times \boldsymbol{B}_{u} \in \mathbb{Q}(\boldsymbol{A}, \boldsymbol{B}) \subseteq \mathrm{K}$. Thus, $\mathrm{K}$ has the JEP, as do its subquasivarieties, in view of the argument - or by heredity of the PSC condition.

The next result allows us to restrict attention to relatively subdirectly irreducible structures when testing a quasivariety for the JEP.

Proposition 4.4. Let $\mathrm{K}$ be a quasivariety, and suppose that, whenever $\boldsymbol{A}, \boldsymbol{B} \in \mathrm{K}_{R S I}$, then there exists $\boldsymbol{C} \in \mathrm{K}$ such that $\boldsymbol{A}$ and $\boldsymbol{B}$ can both be embedded into $\boldsymbol{C}$. Then $\mathrm{K}$ has the JEP.

Proof. Let $\boldsymbol{A}, \boldsymbol{B} \in \mathrm{K}$ be nontrivial. Then

$$
\boldsymbol{A} \in \mathbb{I P}_{\mathrm{S}}\left\{\boldsymbol{A}_{i}: i \in I\right\} \text { and } \boldsymbol{B} \in \mathbb{P}_{\mathrm{S}}\left\{\boldsymbol{B}_{j}: j \in J\right\}
$$

for suitable $\boldsymbol{A}_{i}, \boldsymbol{B}_{j} \in \mathrm{K}_{R S I}$, where $I$ and $J$ are non-empty sets. We may assume that $I \subseteq J$. Fixing $\ell \in I$ and defining $\boldsymbol{A}_{j}=\boldsymbol{A}_{\ell}$ for all $j \in J \backslash I$, we find that $\boldsymbol{A} \in \mathbb{I P}_{\mathrm{S}}\left\{\boldsymbol{A}_{j}: j \in J\right\}$. By assumption, for each $j \in J$, there exists $\boldsymbol{C}_{j} \in \mathrm{K}$ such that $\boldsymbol{A}_{j}, \boldsymbol{B}_{j} \in \mathbb{I S}\left(\boldsymbol{C}_{j}\right)$. Then $\prod_{J} \boldsymbol{A}_{j}$ and $\prod_{J} \boldsymbol{B}_{j}$ both embed into $\boldsymbol{C}:=\prod_{J} \boldsymbol{C}_{j} \in \mathrm{K}$, so $\boldsymbol{A}, \boldsymbol{B} \in \mathbb{I S}(\boldsymbol{C})$.

A quasivariety is said to be finitely generated if it has the form $\mathbb{Q}(\mathrm{K})$ for some finite set $K$ of finite structures. In this case, as $\mathbb{Q}=\mathbb{P P}_{S} \mathbb{S P}_{U}$, we have $\mathbb{Q}(\mathrm{K})_{\mathrm{RSI}} \subseteq \mathbb{I} \mathbb{S}(\mathrm{K})$, where $\mathbb{S}(\mathrm{K})$ is again a finite set of finite structures. Therefore, it follows easily from Proposition 4.4 that the JEP is a decidable property for finitely generated quasivarieties of finite type. 


\section{Algebraic Quasivarieties and the JEP}

From now on, we confine our attention to algebraic quasivarieties.

Given an algebraic quasivariety $\mathrm{K}$ and an algebra $\boldsymbol{A}$ of the same type, the K-congruences (a.k.a. relative congruences) of $\boldsymbol{A}$ are the congruences $\theta$ such that $\boldsymbol{A} / \theta \in \mathrm{K}$. They form an algebraic lattice $\boldsymbol{C o n}_{\mathrm{K}} \boldsymbol{A}$, ordered by inclusion, in which meets are intersections and the compact elements are just the finitely generated K-congruences. We denote by $\Theta_{\mathrm{K}}^{\boldsymbol{A}} Y$ the least K-congruence of $\boldsymbol{A}$ containing a subset $Y$ of $A^{2}$. When $Y=\{\langle a, b\rangle\}$, we write $\Theta_{\mathrm{K}}^{\boldsymbol{A}}(a, b)$ for the principal $\mathrm{K}$-congruence $\Theta_{\mathrm{K}}^{\boldsymbol{A}} Y$. An algebra $\boldsymbol{A} \in \mathrm{K}$ belongs to the class $\mathrm{K}_{R S I}$ [resp. $\mathrm{K}_{R F S I} ; \mathrm{K}_{R S}$ ] iff, in $\boldsymbol{C o n}_{\mathrm{K}} \boldsymbol{A}$, the identity relation $\operatorname{id}_{A}:=\{\langle a, a\rangle: a \in A\}$ is completely meet-irreducible [resp. meetirreducible; a co-atom]. When $\mathrm{K}$ is a variety and $\boldsymbol{A} \in \mathrm{K}$, the congruences and K-congruences of $\boldsymbol{A}$ coincide, so the prefixes and subscripts can be dropped.

An algebra is said to be $n$-generated (where $n$ is a cardinal) if it has a generating subset with at most $n$ elements. An algebraic quasivariety is said to be nontrivial if it has a nontrivial member. In that case, it has a relatively simple member 25, Thm.3.1.8]; for algebraic varieties, this was proved earlier by Magari [37]. On the other hand, even when an algebra is finitely generated (i.e., $n$-generated for some $n \in \omega$ ), it need not have a simple homomorphic image [30, p. 154]. Conditions that guarantee relatively simple homomorphic images are given in the next lemma, which adapts 30 , pp. 153-4] to quasivarieties.

Lemma 5.1. Let $\boldsymbol{A}$ be a nontrivial member of an algebraic quasivariety K.

(i) If the total relation $A^{2}$ is compact in $\boldsymbol{C o n}_{\mathrm{K}} \boldsymbol{A}$, then $\boldsymbol{A}$ has a relatively simple homomorphic image in $\mathrm{K}$.

(ii) If $\boldsymbol{A}$ is finitely generated and of finite type, then $A^{2}$ is compact in $\boldsymbol{C o n}_{\mathrm{K}} \boldsymbol{A}$, so $\boldsymbol{A}$ has a relatively simple homomorphic image in $\mathrm{K}$.

Proof. (ii) If $\perp$ is the least element of an algebraic lattice $\boldsymbol{L}$ and $y \in L \backslash\{\perp\}$ and $y$ is compact in $\boldsymbol{L}$, then $\{x \in L: y \nless x\}$ has a maximal element, by Zorn's Lemma. Setting $\boldsymbol{L}=\boldsymbol{C o n}_{\mathrm{K}} \boldsymbol{A}$ and $y=A^{2}$, we conclude that, under the given assumptions, $\boldsymbol{A}$ has a maximal proper K-congruence $\theta$, whence $\boldsymbol{A} / \theta \in \mathrm{K}$ is $\mathrm{K}$-simple.

(ii) Suppose $\boldsymbol{A}$ is generated by a finite subset $X$ of $A$. Let $Y$ be the union of $X$ and the set of all $f\left(a_{1}, \ldots, a_{n}\right)$ such that $n \in \omega, f$ is a basic $n$ ary operation of $\boldsymbol{A}$ and $a_{1}, \ldots, a_{n} \in X$. Then $A^{2}=\Theta^{\boldsymbol{A}}\left(Y^{2}\right) \subseteq \Theta_{\mathrm{K}}^{\boldsymbol{A}}\left(Y^{2}\right)$. If $\boldsymbol{A}$ has finite type, then $Y^{2}$ is finite, so $A^{2}=\Theta_{\mathrm{K}}^{\boldsymbol{A}}\left(Y^{2}\right)$ is compact in $\boldsymbol{C o n}_{\mathrm{K}} \boldsymbol{A}$, and the last assertion follows from (ii).

Definition 5.2. An algebraic quasivariety will be called a Kollár quasivariety if each of its nontrivial members has no trivial subalgebra.

Clearly, an algebraic quasivariety $\mathrm{K}$ is a Kollár quasivariety if its signature includes two constant symbols that take distinct values in every nontrivial 
member of $\mathrm{K}$. This situation is common in algebraic logic, e.g., every quasivariety of Heyting algebras is a Kollár quasivariety. The result below was proved first for varieties by Kollár [33], hence our nomenclature. Further characterizations of Kollár quasivarieties have been given by Campercholi and Vaggione [9, Prop. 2.3].

Theorem 5.3. (Gorbunov [24, [25, Thm. 2.3.16]) An algebraic quasivariety $\mathrm{K}$ is a Kollár quasivariety iff $A^{2}$ is compact in $\boldsymbol{C o n}_{\mathrm{K}} \boldsymbol{A}$ for every $\boldsymbol{A} \in \mathrm{K}$.

Corollary 5.4. Every nontrivial member of a Kollár quasivariety has a relatively simple homomorphic image.

Proof. This follows from Theorem 5.3 and Lemma 5.1(ii).

Note that an algebra is 0-generated iff it has a distinguished element and no proper subalgebra. An algebra with a distinguished element has a unique 0 -generated subalgebra, which is its smallest subalgebra. These assertions and the first two items of the next result are not always true for structures 2

Proposition 5.5. Let $\mathrm{K}$ be an algebraic quasivariety with the JEP.

(i) ([35]) Any two nontrivial 0-generated members of $\mathrm{K}$ are isomorphic.

(ii) If $\mathrm{K}$ has finite type or is a Kollár quasivariety, then every nontrivial 0-generated member of $\mathrm{K}$ is relatively simple.

(iii) If $\mathrm{K}$ has a constant symbol, then $\mathrm{K}$ is a Kollár quasivariety or every member of $\mathrm{K}$ has a trivial subalgebra.

Proof. (ii) Let $\boldsymbol{A}, \boldsymbol{B} \in \mathrm{K}$ be nontrivial and 0-generated. By the JEP, there exist $\boldsymbol{C} \in \mathrm{K}$ and embeddings $g: \boldsymbol{A} \rightarrow \boldsymbol{C}$ and $h: \boldsymbol{B} \rightarrow \boldsymbol{C}$. As $g[\boldsymbol{A}]$ and $h[\boldsymbol{B}]$ are 0 -generated substructures of $\boldsymbol{C}$, they coincide, so $\left.h^{-1}\right|_{h[B]} \circ g: \boldsymbol{A} \cong \boldsymbol{B}$.

(ii) Let $\boldsymbol{A} \in \mathrm{K}$ be nontrivial and 0-generated. The hypotheses of (iii) imply that $\boldsymbol{A}$ has a homomorphic image $\boldsymbol{B} \in \mathrm{K}_{R S}$, by Corollary 5.4 and Lemma 5.1(iii). Since $\boldsymbol{B}$ is also 0-generated and nontrivial, it is isomorphic to $\boldsymbol{A}$, by (ii), so $\boldsymbol{A}$ is relatively simple.

(iii) Let $c$ be a constant symbol of $\mathrm{K}$. If $\boldsymbol{A} \in \mathrm{K}$ has no trivial subalgebra, then for some basic operation symbol $f$ of $\boldsymbol{A}$, the atomic sentence $f(c, c, \ldots, c) \approx c$ (briefly, $\Phi)$ is false in $\boldsymbol{A}$. In that case, if $\boldsymbol{B} \in \mathrm{K}$ has a proper trivial subalgebra, then $\Phi$ is true in $\boldsymbol{B}$, so $\boldsymbol{A}$ and $\boldsymbol{B}$ have no common extension, contradicting the JEP.

The assumption that $\mathrm{K}$ has a constant symbol cannot be dropped from (iii), even when $\mathrm{K}$ is an algebraic variety (see Example 8.11).

Theorem 5.6. Let $\mathrm{K}$ be a nontrivial Kollár quasivariety with the JEP. Then there is a relatively simple algebra $\boldsymbol{A} \in \mathrm{K}$ such that $\mathbb{I S P}_{\mathrm{U}}(\boldsymbol{A})$ includes every relatively simple member of $\mathrm{K}$.

Consequently, $\mathbb{Q}\left(\mathrm{K}_{R S}\right)=\mathbb{Q}(\boldsymbol{A})$, so $\mathbb{Q}\left(\mathrm{K}_{R S}\right)$ also has the JEP.

\footnotetext{
2 Theorem 5.3 is generalized in 25 to structures having only finitely many basic relations, using an analogue of (relative) congruences that will not be discussed here.
} 
Proof. For any algebra $\boldsymbol{B}$, let $\operatorname{EPS}(\boldsymbol{B})$ denote the set of existential positive sentences that are true in $\boldsymbol{B}$. As $\mathrm{K}$ has the JEP, Theorem 4.2 shows that $\mathrm{K}=\mathbb{Q}(\boldsymbol{C})$ for some $\boldsymbol{C} \in \mathrm{K}$. Since K is nontrivial, so is $\boldsymbol{C}$. By Corollary 5.4. $\boldsymbol{C}$ has a homomorphic image $\boldsymbol{A} \in \mathrm{K}_{R S}$. Observe that

$$
\boldsymbol{A} \models \operatorname{EPS}(\boldsymbol{C}),
$$

as $\boldsymbol{A} \in \mathbb{H}(\boldsymbol{C})$. We claim, moreover, that

$$
\boldsymbol{C} \models \operatorname{EPS}(\boldsymbol{B}) \text {, for every } \boldsymbol{B} \in \mathrm{K}_{R S} \text {. }
$$

Indeed, because $\mathrm{K}=\mathbb{Q}(\boldsymbol{C})=\mathbb{I P}_{\mathrm{S}} \mathbb{S P}_{\mathrm{U}}(\boldsymbol{C})$, we have $\mathrm{K}_{R S} \subseteq \mathrm{K}_{R S I} \subseteq \mathbb{I S P}_{\mathrm{U}}(\boldsymbol{C})$, so $\boldsymbol{C} \in \mathbb{R}_{\mathrm{U}} \mathbb{E} \mathbb{H}(\boldsymbol{B})$ for all $\boldsymbol{B} \in \mathrm{K}_{R S}$. Thus, (3) follows from Corollary 3.2.

Now let $\boldsymbol{B} \in \mathrm{K}_{R S}$. Then $\boldsymbol{A} \models \operatorname{EPS}(\boldsymbol{B})$, by (2) and (3), so there is a homomorphism $h: \boldsymbol{B} \rightarrow \boldsymbol{U}$ for some ultrapower $\boldsymbol{U}$ of $\boldsymbol{A}$, by Theorem 3.1. Since $\boldsymbol{A}$ is nontrivial, so is $\boldsymbol{U}$. Then $h$ is an embedding, by Fact 2.1, as $\mathrm{K}$ is a Kollár quasivariety. Thus, $\boldsymbol{B} \in \mathbb{I S P}_{\mathrm{U}}(\boldsymbol{A})$, as claimed.

This shows that $\mathbb{Q}\left(\mathrm{K}_{R S}\right)=\mathbb{Q}(\boldsymbol{A})$, which has the JEP, by Theorem 4.2 $\quad \square$

Corollary 5.7. Let $\mathrm{K}$ be a nontrivial relatively semisimple Kollár quasivariety with the JEP. Then $\mathrm{K}=\mathbb{Q}(\boldsymbol{A})$ for some relatively simple $\boldsymbol{A} \in \mathrm{K}$.

Proof. This follows from Theorem 5.6, as $\mathrm{K}=\mathbb{Q}\left(\mathrm{K}_{R S I}\right)$ and $\mathrm{K}_{R S I}=\mathrm{K}_{R S} . \quad \square$

Corollary 5.8. Let $\mathrm{K}$ be a nontrivial Kollár quasivariety with the JEP. If the class of all relatively simple members of $\mathrm{K}$ is elementary, then it too has the JEP.

Proof. Let $\boldsymbol{A}, \boldsymbol{B} \in \mathrm{K}_{R S}$. Theorem 5.6 shows that there exist $\boldsymbol{C} \in \mathrm{K}_{R S}$ and $\boldsymbol{U}, \boldsymbol{V} \in \mathbb{P}_{\mathrm{U}}(\boldsymbol{C})$ and embeddings $\boldsymbol{A} \rightarrow \boldsymbol{U}$ and $\boldsymbol{B} \rightarrow \boldsymbol{V}$. Because $\boldsymbol{U}$ and $\boldsymbol{V}$ are elementarily equivalent, they have a common ultrapower $\boldsymbol{W}$, by the Keisler-Shelah Isomorphism Theorem, and of course $\boldsymbol{W} \in \mathrm{K}$. Then $\boldsymbol{A}$ and $\boldsymbol{B}$ both embed into $\boldsymbol{W}$. Moreover, as $\boldsymbol{W}$ is elementarily equivalent to $\boldsymbol{C}$, and as $\mathrm{K}_{R S}$ is elementary, $\boldsymbol{W}$ is relatively simple.

In view of Theorem 4.2 and Corollary 5.7, it is natural to ask whether a quasivariety with the JEP must be generated by a relatively finitely subdirectly irreducible algebra. Even for algebraic PSC varieties, this is not the case, as the next example shows (also see Example 9.7). Here, we require Jónsson's Theorem [29, 31], which asserts that $\mathbb{V}(\mathrm{C})_{F S I} \subseteq \mathbb{H S P}_{\mathrm{U}}(\mathrm{C})$ for any subclass $C$ of a congruence distributive algebraic variety. Recall that every algebra with a lattice reduct generates a congruence distributive variety.

Example 5.9. Let $\mathrm{K}=\mathbb{V}(\boldsymbol{A}, \boldsymbol{B})$, where $\boldsymbol{A}$ and $\boldsymbol{B}$ are the only two non-isomorphic subdirectly irreducible five-element Heyting algebras. Like every variety of Heyting algebras, $\mathrm{K}$ is PSC and therefore has the JEP (see Examples 7.9 below). Suppose $\mathrm{K}=\mathbb{Q}(\boldsymbol{C})$, where $\boldsymbol{C}$ is finitely subdirectly irreducible. By Jónsson's Theorem, $\boldsymbol{C} \in \mathbb{H S P}_{\mathrm{U}}(\boldsymbol{A}, \boldsymbol{B})=\mathbb{H} \mathbb{S}(\boldsymbol{A}, \boldsymbol{B})$ (as $\boldsymbol{A}$ and $\boldsymbol{B}$ are finite), whence $|C| \leq 5$. Now $\boldsymbol{A}$ and $\boldsymbol{B}$ are subdirectly irreducible 
members of $\mathbb{Q}(\boldsymbol{C})=\mathbb{I P}_{\mathrm{S}} \mathbb{S P}_{\mathrm{U}}(\boldsymbol{C})$, so $\boldsymbol{A}, \boldsymbol{B} \in \mathbb{I S P}_{\mathrm{U}}(\boldsymbol{C})=\mathbb{I S}(\boldsymbol{C})$ (as $\boldsymbol{C}$ is finite). Since $|C| \leq|A|,|B|$, this forces $\boldsymbol{A} \cong \boldsymbol{C} \cong \boldsymbol{B}$, a contradiction. Thus, no finitely subdirectly irreducible algebra generates $\mathrm{K}$ as a quasivariety. $\otimes$

An algebraic variety $\mathrm{K}$ is said to have equationally definable principal congruences (EDPC) if there is a finite set $\Sigma$ of pairs of 4-ary terms in its signature such that, whenever $\boldsymbol{A} \in \mathrm{K}$ and $a, b, c, d \in A$, then

$$
\langle c, d\rangle \in \Theta^{\boldsymbol{A}}(a, b) \text { iff }\left(\varphi^{\boldsymbol{A}}(a, b, c, d)=\psi^{\boldsymbol{A}}(a, b, c, d) \text { for all }\langle\varphi, \psi\rangle \in \Sigma\right) .
$$

In this case, $\mathrm{K}$ is congruence distributive and has the congruence extension property $(\mathrm{CEP})$, and its class of simple members is closed under ultraproducts [6].

Theorem 5.10. Let $\mathrm{K}$ be an algebraic variety with EDPC. Then the variety $\mathbb{V}(\boldsymbol{A})$ has the JEP, for every simple algebra $\boldsymbol{A} \in \mathrm{K}$.

Proof. As $\mathrm{K}$ has EDPC, its class of simple members is closed both under $\mathbb{P}_{\mathrm{U}}$ and (by the CEP) under nontrivial subalgebras. So, when $\boldsymbol{A} \in \mathrm{K}$ is simple, the nontrivial members of $\mathbb{H}_{\mathbb{S P}_{\mathrm{U}}}(\boldsymbol{A})$ belong to $\mathbb{I S P}_{\mathrm{U}}(\boldsymbol{A})$. In this case, by Jónsson's Theorem, $\mathbb{V}(\boldsymbol{A})=\mathbb{Q}(\boldsymbol{A})$, which has the JEP, by Theorem 4.2, $\square$

The JEP has a syntactic meaning in algebraic logic, which we recount below in the context of algebraic quasivarieties. For a set $\Gamma$ of formal equations, we denote by $\operatorname{Var}(\Gamma)$ the set of all variables $x$ such that $x$ occurs in at least one member of $\Gamma$.

Definition 5.11. An algebraic quasivariety $\mathrm{K}$ is said to respect the relevance principle if the following is true whenever $\Gamma \cup \Delta \cup\{\varphi \approx \psi\}$ is a finite set of equations, with $\operatorname{Var}(\Delta) \cap \operatorname{Var}(\Gamma \cup\{\varphi \approx \psi\})=\emptyset$, and $\Delta$ is consistent over $\mathrm{K}$ (i.e., there exist terms $\alpha, \beta$ such that $\mathrm{K} \not \forall(\& \Delta) \Longrightarrow \alpha \approx \beta)$ :

$$
\text { if } \mathrm{K} \models(\&(\Gamma \cup \Delta)) \Longrightarrow \varphi \approx \psi \text {, then } \mathrm{K} \models(\& \Gamma) \Longrightarrow \varphi \approx \psi \text {. }
$$

Theorem 5.12. An algebraic quasivariety has the JEP iff it respects the relevance principle.

This is an algebraic analogue of the Eos-Suszko Theorem [36, p. 182], which concerns sentential deductive systems, i.e., substitution-invariant consequence relations over terms in an algebraic signature. (Variants of the Łos-Suszko Theorem for special families of deductive systems are discussed in [2, 22, 32, 38, 39, 65].)

The forward implication of Theorem 5.12 follows from item (iii) after Theorem 4.2. because a quasi-equation is logically equivalent to a disjunction (the disjuncts being its conclusion and the negations of its premises). Conversely, if $\boldsymbol{A}, \boldsymbol{B}$ are disjoint nontrivial members of an algebraic quasivariety $\mathrm{K}$, then the respective identity functions on $A$ and $B$ extend to surjective homomorphisms $\pi_{A}: \boldsymbol{F}_{\mathrm{K}}(A) \rightarrow \boldsymbol{A}$ and $\pi_{B}: \boldsymbol{F}_{\mathrm{K}}(B) \rightarrow \boldsymbol{B}$ (where $\boldsymbol{F}_{\mathrm{K}}(X)$ denotes a member of $\mathrm{K}$ that is $\mathrm{K}$-free over $X)$. In $\boldsymbol{F}:=\boldsymbol{F}_{\mathrm{K}}(A \cup B)$, let $\theta$ be the 
K-congruence generated by the union of the kernels of $\pi_{A}$ and $\pi_{B}$, and let $\boldsymbol{C}=\boldsymbol{F} / \theta$, so $\boldsymbol{C} \in \mathrm{K}$. The map $a \mapsto a / \theta$ [resp. $b \mapsto b / \theta$ ] is a homomorphism from $\boldsymbol{A}[$ resp. $\boldsymbol{B}]$ into $\boldsymbol{C}$. Its injectivity follows from the relevance principle for K, using the algebraicity of the lattice $\boldsymbol{C o n}_{\mathrm{K}} \boldsymbol{F}$.

Remark 5.13. For a class $K$ of similar algebras, let $\mathbb{U}(K)$ be the class of all algebras $\boldsymbol{B}$ such that every $|\operatorname{Var}|$-generated subalgebra of $\boldsymbol{B}$ belongs to $\mathrm{K}$. In general, $\mathbb{U I S P}(\mathrm{K}) \subseteq \mathbb{Q}(\mathrm{K})$, and the two need not be equal. If $\mathrm{K}$ is a quasivariety with the JEP, however, then there exists $\boldsymbol{A} \in \mathrm{K}$ such that $\mathrm{K}=\mathbb{U I S P}(\boldsymbol{A})$, by item (ii) after Theorem 4.2, because the $|\operatorname{Var}|$-generated members of $\mathrm{K}$ form a set, up to isomorphism. This means that, even if we allowed quasi-equations (over Var) to have infinitely many premises, their validity in $\boldsymbol{A}$ would entail their validity throughout K. In fact, if we generalized Definition 5.11 to such formulas, then Theorem 5.12 would remain true.

\section{Structural Completeness}

In a given algebraic signature, a substitution is an endomorphism of the absolutely free algebra (a.k.a. the term algebra) generated by Var. For an algebraic quasivariety $\mathrm{K}$ and a cardinal $m$, recall that the $\mathrm{K}$-free $m$ generated algebra $\boldsymbol{F}_{\mathrm{K}}(m) \in \mathrm{K}$ exists iff $m>0$ or $\mathrm{K}$ has a constant symbol. Of course, every algebraic variety $\mathrm{K}$ is generated as such by its free denumerably generated algebra, i.e., $\mathrm{K}=\mathbb{V}\left(\boldsymbol{F}_{\mathrm{K}}\left(\aleph_{0}\right)\right)$, but $\mathrm{K}$ need not coincide with the quasivariety $\mathbb{Q}\left(\boldsymbol{F}_{\mathrm{K}}\left(\aleph_{0}\right)\right)$ (which has the JEP, by Theorem 4.2).

Theorem 6.1. ([3, Prop. 2.3]) The following conditions on an algebraic quasivariety $\mathrm{K}$ are equivalent.

(i) $\mathrm{K}=\mathbb{Q}\left(\boldsymbol{F}_{\mathrm{K}}\left(\aleph_{0}\right)\right)$.

(ii) Whenever $\mathrm{K}^{\prime}$ is a proper subquasivariety of $\mathrm{K}$, then $\mathrm{K}^{\prime}$ and $\mathrm{K}$ generate distinct varieties, i.e., $\mathbb{H}\left(\mathrm{K}^{\prime}\right) \subsetneq \mathbb{H}(\mathrm{K})$.

(iii) For each quasi-equation $\left(\varphi_{1} \approx \psi_{1} \& \cdots \& \varphi_{n} \approx \psi_{n}\right) \Longrightarrow \varphi \approx \psi$ that is invalid in (some member of) $\mathrm{K}$, there exists a substitution $h$ such that $\mathrm{K} \models h\left(\varphi_{i}\right) \approx h\left(\psi_{i}\right)$ for $i=1, \ldots, n$, but $\mathrm{K} \not \models h(\varphi) \approx h(\psi)$.

Definition 6.2. An algebraic quasivariety $\mathrm{K}$ is said to be structurally complete (SC) if it satisfies the equivalent conditions of Theorem 6.1. It is hereditarily structurally complete (HSC) if, in addition, its subquasivarieties are all SC.

In particular, an algebraic variety $\mathrm{K}$ is $\mathrm{SC}$ iff each of its proper subquasivarieties generates a proper subvariety of $\mathrm{K}$; it is $\mathrm{HSC}$ iff its subquasivarieties are all varieties. These notions have logical origins: the algebraic counterpart of an algebraizable deductive system $\vdash$ (in the sense of [7]) is SC iff every proper extension of $\vdash$ has some new theorem - as opposed to having nothing but new rules of derivation; it is HSC iff every extension of $\vdash$ is axiomatic (see for instance [51, 53, 57]). The terminology originates in [52]. 


\section{Passive Structural Completeness}

Every SC algebraic quasivariety $\mathrm{K}$ is PSC in the sense of Definition 3.4. Indeed, if $\boldsymbol{A}, \boldsymbol{B} \in \mathrm{K}$ are nontrivial, then $\boldsymbol{A}$ has a homomorphic image $\boldsymbol{C} \in \mathrm{K}_{R S I}$, while $\boldsymbol{B}$ is an extension of a 1-generated homomorphic image of $\boldsymbol{F}_{\mathrm{K}}\left(\aleph_{0}\right)$, so it suffices to show that $\boldsymbol{F}_{\mathrm{K}}\left(\aleph_{0}\right)$ satisfies the existential positive sentences that are true in $\boldsymbol{C}$. This is indeed the case, by Theorem 3.1, as $\boldsymbol{C} \in \mathbb{I S P}_{\mathrm{U}}\left(\boldsymbol{F}_{\mathrm{K}}\left(\aleph_{0}\right)\right)$ (because $\mathrm{K}=\mathbb{I P}_{\mathrm{S}} \mathbb{S P}_{\mathrm{U}}\left(\boldsymbol{F}_{\mathrm{K}}\left(\aleph_{0}\right)\right)$, by Theorem 6.1(1) ). Alternatively, condition (iii) of Theorem 6.1 clearly entails the characterization of passive structural completeness in Theorem 7.2 below.

The above argument and Theorem 4.3 establish the implications

$$
\mathrm{HSC} \Longrightarrow \mathrm{SC} \Longrightarrow \mathrm{PSC} \Longrightarrow \mathrm{JEP}
$$

none of which is reversible. A variety of lattices that is SC but not HSC is exhibited in [3, Ex. 2.14.4]. It is well known (and follows, for instance, from [47]) that the variety of Heyting algebras is not SC, but it is PSC (see Examples (7.9). An algebraic variety with the JEP that is not PSC will be pointed out in Example 8.11.

Definition 7.1. A set $\Gamma$ of equations in the signature of an algebraic quasivariety $\mathrm{K}$ is said to be unifiable over $\mathrm{K}$ if there is a substitution $h$ such that $\mathrm{K} \models h(\varphi) \approx h(\psi)$ for every equation $\varphi \approx \psi$ from $\Gamma$. A quasi-equation

$$
\left(\varphi_{1} \approx \psi_{1} \& \cdots \& \varphi_{n} \approx \psi_{n}\right) \Longrightarrow \varphi \approx \psi
$$

in the same signature is said to be active [resp. passive] over $\mathrm{K}$ if its set of premises $\left\{\varphi_{i} \approx \psi_{i}: i=1, \ldots, n\right\}$ is [resp. is not] unifiable over $\mathrm{K}$.

The next result amplifies the logical meaning of passive structural completeness. (It strengthens an earlier finding of Bergman [3, Thm. 2.7].)

Theorem 7.2. (Wroński [68, Fact 2, p.68]) An algebraic quasivariety $\mathrm{K}$ is $P S C$ iff every quasi-equation that is passive over $\mathrm{K}$ is valid in (all members of) $\mathrm{K}$.

Theorem 7.2 motivates the 'passive' terminology used above, which is adapted from [11. (A complementary demand, now called 'active structural completeness' and analysed in [17, asks that condition (iii) of Theorem 6.1 should hold for all active quasi-equations; also see 60. Computational aspects of [active] structural completeness are addressed in [16, 43, 64].)

Obviously, a quasi-equation is passive over an algebraic quasivariety $\mathrm{K}$ iff it is passive over the variety $\mathbb{V}(\mathrm{K})$. It may happen that $\mathrm{K}$ is $\mathrm{PSC}$ for the vacuous reason that no quasi-equation is passive over $\mathrm{K}$ (as applies, for instance, to every quasivariety of lattices). The next result and its corollary decode this case in model-theoretic terms. Their itemized conditions persist, of course, under varietal generation, unlike passive structural completeness itself. 
Theorem 7.3. Let $\mathrm{K}$ be an algebraic quasivariety. Then the following conditions are equivalent.

(i) No quasi-equation is passive over $\mathrm{K}$ (i.e., every finite set of equations in the signature of $\mathrm{K}$ is unifiable over $\mathrm{K}$ ).

(ii) $\mathrm{K}$ is $P S C$ and is either trivial or not a Kollár quasivariety.

(iii) Every member of $\mathrm{K}$ has an ultrapower with a trivial subalgebra.

(iv) $\boldsymbol{F}_{\mathrm{K}}(1)$ has an ultrapower with a trivial subalgebra.

Proof. (ii) $\Rightarrow$ (iii): Certainly, $\mathrm{K}$ is PSC, by (ii) and Theorem 7.2. If $\boldsymbol{F}_{\mathrm{K}}(1)$ is trivial, then every member of $\mathrm{K}$ has a trivial subalgebra, so we may assume that $\boldsymbol{F}_{\mathrm{K}}(1)$ is nontrivial.

Let $\Sigma$ be the set of all existential positive sentences in the first-order signature of $\mathrm{K}$, and let $\left\{f_{1}, \ldots, f_{n}\right\}$ be any finite set of basic operation symbols of $\mathrm{K}$. By (ii), the equations $f_{i}(x, \ldots, x) \approx x(i=1, \ldots, n)$ are unifiable, i.e., there is a term $\varphi$ such that $\mathrm{K} \models f_{i}(\varphi, \ldots, \varphi) \approx \varphi$ for $i=1, \ldots, n$. Identifying variables, we see that $\varphi$ may be chosen unary, whence

$$
\boldsymbol{F}_{\mathrm{K}}(1) \models \exists x\left(x \approx f_{1}(x, \ldots, x) \approx \cdots \approx f_{n}(x, \ldots, x)\right) .
$$

As $\left\{f_{1}, \ldots, f_{n}\right\}$ was arbitrary, this implies that $\boldsymbol{F}_{\mathrm{K}}(1) \models \Sigma$. Let $\boldsymbol{C} \in \mathrm{K}$ be trivial. Of course, $\Sigma$ is the set of all existential positive sentences that hold in $\boldsymbol{C}$, so by Theorem 3.1, $\boldsymbol{C}$ can be mapped homomorphically into an ultrapower $\boldsymbol{U}$ of $\boldsymbol{F}_{\mathrm{K}}(1)$, i.e., $\boldsymbol{U}$ has a trivial subalgebra. Now $\boldsymbol{U}$ is nontrivial (because $\boldsymbol{F}_{\mathrm{K}}(1) \in \mathbb{I} \mathbb{S}(\boldsymbol{U})$ ), so $\mathrm{K}$ is not a Kollár quasivariety.

(iii) $\Rightarrow$ (iii): Let $\boldsymbol{A} \in \mathrm{K}$. We may assume that $\mathrm{K}$ is nontrivial (otherwise, (iii) is immediate). Then, by (iii), some nontrivial $\boldsymbol{B} \in \mathrm{K}$ has a trivial subalgebra $\boldsymbol{C}$, and $\mathrm{K}$ is PSC, so $\boldsymbol{A}$ satisfies all existential positive sentences that are true in $\boldsymbol{B}$. Thus, there is a homomorphism $h: \boldsymbol{B} \rightarrow \boldsymbol{U}$ for some ultrapower $\boldsymbol{U}$ of $\boldsymbol{A}$, by Theorem [3.1, and $h[\boldsymbol{C}]$ is a trivial subalgebra of $\boldsymbol{U}$.

(iii) $\Rightarrow$ (iv) is immediate, since $\boldsymbol{F}_{\mathrm{K}}(1) \in \mathrm{K}$.

(iv) $\Rightarrow$ (ii): Let $\boldsymbol{U} \in \mathbb{P}_{\mathrm{U}}\left(\boldsymbol{F}_{\mathrm{K}}(1)\right)$, where $\boldsymbol{U}$ has a trivial subalgebra. Then, for any finite set $\Gamma$ of equations in the signature of $\mathrm{K}$, the sentence $\exists \bar{x}(\& \Gamma)$ is true in $\boldsymbol{U}$, so it is true in $\boldsymbol{F}_{\mathrm{K}}(1)$. Therefore, $\Gamma$ is unifiable over $\mathrm{K}$.

Corollary 7.4. Let $\mathrm{K}$ be an algebraic quasivariety, either of finite type or whose free 1-generated algebra is finite. Then no quasi-equation is passive over $\mathrm{K}$ iff every member of $\mathrm{K}$ has a trivial subalgebra.

Proof. Sufficiency follows from Theorem 7.3. Conversely, suppose that no quasi-equation is passive over K. Then some ultrapower $\boldsymbol{A}$ of $\boldsymbol{F}_{\mathrm{K}}(1)$ has a trivial subalgebra, again by Theorem 7.3. It clearly suffices to show that $\boldsymbol{F}_{\mathrm{K}}(1)$ has a trivial subalgebra. If $\boldsymbol{F}_{\mathrm{K}}(1)$ is finite, then it is isomorphic to $\boldsymbol{A}$, and we are done. If the signature of $\mathrm{K}$ is finite then, for its models, the property of having a trivial subalgebra is expressed by an existential positive sentence (which persists, of course, in ultraroots). In that case, $\boldsymbol{F}_{\mathrm{K}}(1)$ has a trivial subalgebra, because $\boldsymbol{A}$ does. 
In general, however, the ultrapowers in Theorem 7.3 cannot be eliminated, because of the next example.

Example 7.5. For $n \in \mathbb{N}=\{1,2,3, \ldots\}$, let $f_{n}: \mathbb{N} \rightarrow \mathbb{N}$ be the function such that $f_{n}(m)=m+n$ for $m=1, \ldots, n$ and $f_{n}(m)=m$ whenever $n<m \in \mathbb{N}$. Let $\boldsymbol{A}$ be the algebra with universe $\mathbb{N}$, whose set of basic operations is $\left\{f_{n}: n \in \mathbb{N}\right\}$, and let $\mathrm{K}=\mathbb{V}(\boldsymbol{A})$. In this signature, every term that is not a variable has the form $f_{i_{1}} \ldots f_{i_{k}}(x)$ for some $i_{1}, \ldots, i_{k} \in \mathbb{N}$. Therefore, since $\boldsymbol{A}$ generates K, every finite set of equations can be unified over $\mathrm{K}$ by substituting $f_{r}(x)$ for every variable, where $r$ is sufficiently large. Thus, no quasi-equation is passive over K, but $\boldsymbol{A}$ is a nontrivial member of $\mathrm{K}$ that has no trivial subalgebra. (For each non-principal ultrafilter $\mathcal{U}$ over $\mathbb{N}$, the ultrapower $\boldsymbol{A}^{\mathbb{N}} / \mathcal{U}$ has a trivial subuniverse, viz. $\{\langle 1,2,3, \ldots\rangle / \mathcal{U}\}$.)

Recall that an algebra $\boldsymbol{A}$ is said to be a retract of an algebra $\boldsymbol{B}$ if there are homomorphisms $g: \boldsymbol{A} \rightarrow \boldsymbol{B}$ and $h: \boldsymbol{B} \rightarrow \boldsymbol{A}$ such that $h \circ g$ is the identity function $\operatorname{id}_{A}$ on $A$. This forces $g$ to be injective and $h$ surjective.

The next result identifies the PSC quasivarieties of finite type containing at least one finite nontrivial algebra.

Theorem 7.6. Let $\mathrm{K}$ be an algebraic quasivariety of finite type, with a finite nontrivial member. Then the following conditions are equivalent.

(i) $\mathrm{K}$ is $P S C$.

(ii) The nontrivial members of $\mathrm{K}$ have a common retract.

(iii) Each nontrivial member of $\mathrm{K}$ can be mapped homomorphically into every member of $\mathrm{K}$.

In this case, the nontrivial members of $\mathrm{K}$ have a finite common retract that has no nontrivial proper subalgebra and is either trivial or relatively simple.

Moreover, when $\mathrm{K}$ is PSC, its nontrivial members have at most one nontrivial common retract, and they have at most one 0-generated common retract (up to isomorphism).

Proof. By assumption, $\mathrm{K}$ has a finite nontrivial member, and that algebra has a relatively simple (finite nontrivial) homomorphic image $\boldsymbol{A} \in \mathrm{K}$.

(ii) $\Rightarrow$ (iii): Possession of a trivial subalgebra is expressible, over $\mathrm{K}$, by an existential positive sentence, because $\mathrm{K}$ has finite type. Therefore, since $\mathrm{K}$ is $\mathrm{PSC}$, if some nontrivial member of $\mathrm{K}$ has a trivial subalgebra, then so does every member of $\mathrm{K}$. In that case, every member of $\mathrm{K}$ has a trivial retract.

We may therefore assume that $\mathrm{K}$ is a Kollár quasivariety. In particular, $\boldsymbol{A}$ has no trivial subalgebra. To complete the proof of (iii), we shall show that $\boldsymbol{A}$ is a retract of every nontrivial member of $\mathrm{K}$.

Accordingly, let $\boldsymbol{B} \in \mathrm{K}$ be nontrivial, so $\boldsymbol{B}$ has no trivial subalgebra. Since $\boldsymbol{A}$ is finite and of finite type, there is an existential positive sentence $\Phi$ such that an algebra in the signature of $\mathrm{K}$ satisfies $\Phi$ iff it has a subalgebra that is a homomorphic image of $\boldsymbol{A}$. As $\Phi$ is true in $\boldsymbol{A}$, it is true in $\boldsymbol{B}$, 
because $\mathrm{K}$ is PSC (and since $\boldsymbol{A}$ and $\boldsymbol{B}$ are nontrivial). Therefore, there is a homomorphism $g: \boldsymbol{A} \rightarrow \boldsymbol{B}$. As $\boldsymbol{A}$ is relatively simple and $\boldsymbol{B}$ has no trivial subalgebra, $g$ is an embedding, by Fact 2.1. Moreover, since $\mathrm{K}$ is PSC, there is a homomorphism $h$ from $\boldsymbol{B}$ into an ultrapower of $\boldsymbol{A}$, but $\boldsymbol{A}$ is finite, so $h: \boldsymbol{B} \rightarrow \boldsymbol{A}$. Thus, $h \circ g$ is an endomorphism of $\boldsymbol{A}$.

Because $\boldsymbol{A}$ has no trivial subalgebra, the argument for the injectivity of $g$ applies equally to $h \circ g$. Then, since $h \circ g$ is an injection from the finite set $A$ to itself, it is surjective, i.e., $h \circ g$ is an automorphism of $\boldsymbol{A}$.

As the automorphism group of $\boldsymbol{A}$ is finite, $(h \circ g)^{n+1}=\mathrm{id}_{A}$ for some $n \in \omega$. Then, for the homomorphism $k:=g \circ(h \circ g)^{n}: \boldsymbol{A} \rightarrow \boldsymbol{B}$, we have $h \circ k=(h \circ g)^{n+1}=\mathrm{id}_{A}$. Thus, $\boldsymbol{A}$ is a retract of $\boldsymbol{B}$, as claimed.

We have shown that a finite common retract $\boldsymbol{A}^{\prime}$ of the nontrivial members of $\mathrm{K}$ exists and can be chosen relatively simple or trivial. Being finite, $\boldsymbol{A}^{\prime}$ cannot be a retract of a proper subalgebra of itself, so it has no such nontrivial subalgebra. In particular, if $\boldsymbol{A}^{\prime}$ is nontrivial, then it is isomorphic to any other nontrivial common retract of the nontrivial members of K. Consequently, if $\boldsymbol{A}^{\prime}$ is 0-generated, then it is isomorphic to any other common retract of the nontrivial members of $\mathrm{K}$, because it is either trivial or has no trivial subalgebra.

(iii) $\Rightarrow$ (iii): Let $\boldsymbol{C}, \boldsymbol{D} \in \mathrm{K}$, where $\boldsymbol{C}$ is nontrivial. We may assume that $\boldsymbol{D}$ is nontrivial, so there is a common retract $\boldsymbol{A}$ of $\boldsymbol{C}, \boldsymbol{D}$, by (iii). Then there exist a surjective homomorphism $\boldsymbol{C} \rightarrow \boldsymbol{A}$ and an embedding $\boldsymbol{A} \rightarrow \boldsymbol{D}$, whose composition is a homomorphism $\boldsymbol{C} \rightarrow \boldsymbol{D}$.

(iii) $\Rightarrow$ (ii): Let $\boldsymbol{C}, \boldsymbol{D} \in \mathrm{K}$ be nontrivial. By (iii), $\boldsymbol{C}$ can be mapped homomorphically into (an ultrapower of) $\boldsymbol{D}$, so $\mathrm{K}$ is PSC.

Note 7.7. In Theorem 7.6, the finiteness of the signature and the presence of a finite nontrivial algebra in $\mathrm{K}$ are needed only for the implication (ii) $\Rightarrow$ (iii).

It follows easily from Theorem 7.6 (iii) that passive structural completeness is a decidable property for finitely generated algebraic quasivarieties of finite type. Also, 7.6(iii) amounts to the demand that each nontrivial member of $\mathrm{K}$ is a retract of its direct product with any member of $\mathrm{K}$.

Corollary 7.8. Let $\mathrm{K}$ be a PSC Kollár quasivariety of finite type, with a finite nontrivial member. Then $\mathrm{K}$ has a unique relatively simple member (up to isomorphism), and that algebra is a finite common retract of the nontrivial members of $\mathrm{K}$.

Proof. This follows from Theorem 7.6, because a relatively simple member of a Kollár quasivariety is isomorphic to each of its retracts (by Fact 2.1). $\square$

Examples 7.9. It follows from Theorem 7.6 that every variety consisting of groups or of Heyting algebras is PSC (and therefore has the JEP, by Theorem 4.3). Indeed, every nontrivial group has both a trivial retract and a subgroup with a finite nontrivial homomorphic image, while the twoelement Boolean algebra is a retract of every nontrivial Heyting algebra. The 
class of all distributive lattices is a PSC variety whose nontrivial members have both a trivial and a nontrivial common retract, the latter being the two-element lattice. In Corollary [7.8, we cannot drop the demand that $\mathrm{K}$ be a Kollár quasivariety, as the variety of abelian groups satisfies the other hypotheses, but includes all the simple groups $\mathbb{Z}_{p}$ ( $p$ a positive prime). $\otimes$

Remark 7.10. A 0-generated algebra $\boldsymbol{A}$ is a retract of an algebra $\boldsymbol{B}$ if there exist homomorphisms $g: \boldsymbol{A} \rightarrow \boldsymbol{B}$ and $h: \boldsymbol{B} \rightarrow \boldsymbol{A}$. For in this case, every element of $A$ has the form $\alpha^{\boldsymbol{A}}\left(c_{1}, \ldots, c_{n}\right)$ for some term $\alpha$ and some distinguished elements $c_{i} \in A$, whence $h \circ g=\mathrm{id}_{A}$, because homomorphisms preserve distinguished elements (and respect terms).

Notation. For an algebraic quasivariety $\mathrm{K}$, with $\boldsymbol{A} \in \mathrm{K}$, we define

$$
\operatorname{Ret}(\mathrm{K}, \boldsymbol{A})=\{\boldsymbol{B} \in \mathrm{K}: \boldsymbol{B} \text { is trivial or } \boldsymbol{A} \text { is a retract of } \boldsymbol{B}\} \text {. }
$$

Theorem 7.11. Let $\mathrm{K}$ be an algebraic quasivariety of finite type, and $\boldsymbol{A} \in \mathrm{K}$ a finite 0-generated algebra.

(i) $\operatorname{Ret}(\mathrm{K}, \boldsymbol{A})$ is a PSC quasivariety.

(ii) If $\boldsymbol{A}$ is nontrivial or $\mathrm{K}$ is not a Kollár quasivariety, then $\operatorname{Ret}(\mathrm{K}, \boldsymbol{A})$ is a maximal PSC subquasivariety of $\mathrm{K}$.

(iii) If $\mathrm{K}^{\prime}$ is a maximal $P S C$ subquasivariety of $\mathrm{K}$, and if $\boldsymbol{B}^{\prime} \in \mathrm{K}^{\prime}$ is finite and nontrivial, then $\mathrm{K}^{\prime}=\operatorname{Ret}\left(\mathrm{K}, \boldsymbol{A}^{\prime}\right)$, where $\boldsymbol{A}^{\prime}$ is the 0-generated subalgebra of $\boldsymbol{B}^{\prime}$.

(iv) Every PSC subquasivariety of $\mathrm{K}$ that has a finite nontrivial member is contained in just one maximal PSC subquasivariety of $\mathrm{K}$.

Proof. Let $\mathrm{L}=\operatorname{Ret}(\mathrm{K}, \boldsymbol{A})$.

(ii) It suffices, by Note 7.7, to show that $L$ is a quasivariety. As $L$ is isomorphically closed, we must show that it is closed under $\mathbb{S}, \mathbb{P}$ and $\mathbb{P}_{U}$, bearing Remark 7.10 in mind. If $\boldsymbol{C} \in \mathbb{S}(\boldsymbol{B})$ and $h: \boldsymbol{B} \rightarrow \boldsymbol{A}$ is a homomorphism, then so is $\left.h\right|_{C}: \boldsymbol{C} \rightarrow \boldsymbol{A}$, while any embedding $\boldsymbol{A} \rightarrow \boldsymbol{B}$ maps into $\boldsymbol{C}$, as $\boldsymbol{A}$ is 0 -generated. Thus, $\mathrm{L}$ is closed under $\mathbb{S}$. Let $\left\{\boldsymbol{B}_{i}: i \in I\right\}$ be a subfamily of $\mathrm{L}$, where, without loss of generality, $I \neq \emptyset$. For any $j \in I$, the projection $\prod_{i \in I} \boldsymbol{B}_{i} \rightarrow \boldsymbol{B}_{j}$ can be composed with a homomorphism $\boldsymbol{B}_{j} \rightarrow \boldsymbol{A}$, while $\boldsymbol{A}$ embeds diagonally into $\prod_{i \in I} \boldsymbol{B}_{i}$, so $\prod_{i \in I} \boldsymbol{B}_{i} \in \mathrm{L}$. As $\boldsymbol{A}$ is finite, it is isomorphic to each of its ultrapowers, so because $\mathbb{P}_{\mathrm{U}} \mathbb{H}\left(\mathrm{L}^{\prime}\right) \subseteq \mathbb{H}_{\mathrm{U}}\left(\mathrm{L}^{\prime}\right)$ for any class $\mathrm{L}^{\prime}$ of similar algebras, it follows that every ultraproduct of $\left\{\boldsymbol{B}_{i}: i \in I\right\}$ can be mapped homomorphically to $\boldsymbol{A}$. Also, as $\boldsymbol{A}$ is finite and of finite type, the attribute of having a subalgebra isomorphic to $\boldsymbol{A}$ is first order-definable and therefore persists in ultraproducts. Thus, $\mathrm{L}$ is closed under $\mathbb{P}$ and $\mathbb{P}_{\mathrm{U}}$.

(ii) Suppose $\mathrm{L} \subseteq \mathrm{K}^{\prime} \subseteq \mathrm{K}$, where $\mathrm{K}^{\prime}$ is a PSC quasivariety. Then $\boldsymbol{A} \in \mathrm{K}^{\prime}$. If $\boldsymbol{A}$ is nontrivial, then Theorem 7.6 applies to $\mathrm{K}^{\prime}$ (because $\boldsymbol{A}$ is finite) and it shows that, for every nontrivial $\boldsymbol{C} \in \mathrm{K}^{\prime}$, there are homomorphisms $\boldsymbol{A} \rightarrow \boldsymbol{C}$ and $\boldsymbol{C} \rightarrow \boldsymbol{A}$ (as $\mathrm{K}^{\prime}$ is PSC). In this case $\mathrm{K}^{\prime} \subseteq \mathrm{L}$, by Remark 7.10 (as $\boldsymbol{A}$ is 0 -generated). We may therefore assume that $\boldsymbol{A}$ is trivial. Now suppose $\mathrm{K}$ is not a Kollár quasivariety. Then $\boldsymbol{A}$ embeds into some nontrivial $\boldsymbol{B} \in \mathrm{K}$, 
whence $\boldsymbol{B} \in \mathrm{L}$, and so $\boldsymbol{B} \in \mathrm{K}^{\prime}$. Thus, $\mathrm{K}^{\prime}$ is not a Kollár quasivariety. Then $\mathrm{K}^{\prime} \subseteq \mathrm{L}$, by Proposition 5.5(iii) and Theorem 4.3 .

(iii) Let $\mathrm{K}^{\prime}, \boldsymbol{B}^{\prime}, \boldsymbol{A}^{\prime}$ be as described. By (ii), it is enough to show that $\mathrm{K}^{\prime} \subseteq \operatorname{Ret}\left(\mathrm{K}, \boldsymbol{A}^{\prime}\right)$. This will be true if every member of $\mathrm{K}^{\prime}$ has a trivial subalgebra (in which case $\boldsymbol{A}^{\prime}$ is trivial). We may therefore assume, by Proposition 5.5(iii) and Theorem 4.3, that $\mathrm{K}^{\prime}$ is a Kollár quasivariety (as $\mathrm{K}^{\prime}$ is PSC). Then $\boldsymbol{A}^{\prime}$ is nontrivial, so it is $\mathrm{K}^{\prime}$-simple, by Proposition 5.5(iii). Thus, $\mathrm{K}^{\prime} \subseteq \operatorname{Ret}\left(\mathrm{K}, \boldsymbol{A}^{\prime}\right)$, by Corollary 7.8 ,

(iv) follows from Theorem 7.6, together with (ii)-(iii).

A quasivariety is said to be minimal if it is nontrivial and has no nontrivial proper subquasivariety. If we say that a variety is minimal (without further qualification), we mean that it is nontrivial and has no nontrivial proper subvariety. When we mean instead that it is minimal as a quasivariety, we shall say so explicitly, thereby avoiding ambiguity. Obviously, any minimal algebraic quasivariety is HSC, and hence (P)SC. Recall that if a (quasi)variety is finitely generated then it is locally finite, i.e., its finitely generated members are finite [8, Thm. II.10.16].

Theorem 7.12. ([4]) Every locally finite congruence modular minimal algebraic variety is also minimal as a quasivariety (and therefore $H S C$ ).

Theorem 7.13. A relatively semisimple algebraic quasivariety $\mathrm{K}$ is $P S C$ iff it is a minimal quasivariety or has no passive quasi-equation.

Proof. Sufficiency is obvious. Conversely, let $\mathrm{K}$ be PSC and suppose that some quasi-equation is passive over $\mathrm{K}$. Then $\mathrm{K}$ is a nontrivial Kollár quasivariety, by Theorem 7.3 . Let $\boldsymbol{A} \in \mathrm{K}$ be nontrivial. As $\mathrm{K}$ is relatively semisimple, its minimality will follow if we can show that $\mathrm{K}_{R S} \subseteq \mathbb{Q}(\boldsymbol{A})$, so let $\boldsymbol{B} \in \mathrm{K}_{R S}$. Since $\boldsymbol{B}$ is nontrivial and $\mathrm{K}$ is PSC, there is a homomorphism $h$ from $\boldsymbol{B}$ into an ultrapower $\boldsymbol{C}$ of $\boldsymbol{A}$. Of course, $\boldsymbol{C}$ is also nontrivial, so $h$ is an embedding, by Fact 2.1 , because $\mathrm{K}$ is a Kollár quasivariety. Thus, $\boldsymbol{B} \in \mathbb{I S}(\boldsymbol{C}) \subseteq \mathbb{I S P}_{\mathrm{U}}(\boldsymbol{A}) \subseteq \mathbb{Q}(\boldsymbol{A})$, as required.

The proof of Theorem 7.13 yields the following.

Corollary 7.14. If a relatively semisimple algebraic quasivariety with a passive quasi-equation is PSC, then it is both a Kollár quasivariety and a minimal quasivariety (and is therefore $H S C$ ).

Heyting and Brouwerian algebras model intuitionistic propositional logic and its positive fragment, respectively (see Definition 10.1). We have noted that all varieties of Heyting algebras are PSC; the same applies to Brouwerian algebras, as they have trivial retracts. Citkin has determined the HSC varieties of Heyting algebras [13] and of Brouwerian algebras 14] (also see Theorem 10.8 below). An analogous result for modal K4-algebras was proved by Rybakov [59, 60]. Certain fragments of intuitionistic logic are 
modeled by HSC varieties [46, 55, 56, 67] (also see [12]); for the case of relevance logic, see [63] and [51, Sec. 6,9]. The next two sections of the present paper focus on the algebras of relevance logic (in its full signature), and their completeness properties.

\section{De Morgan Monoids: A Case Study}

De Morgan monoids were introduced by Dunn [15, 45]. In the terminology of [7], they constitute the equivalent algebraic semantics for the principal relevance logic $\mathbf{R}^{\mathbf{t}}$ of [1], and the quasivarieties of De Morgan monoids algebraize the extensions of $\mathbf{R}^{\mathbf{t}}$ by new axioms and/or inference rules. There is a transparent lattice anti-isomorphism from the subquasivarieties to the extensions, with subvarieties corresponding to purely axiomatic extensions. Accordingly, in [48, 49] we undertook an investigation of the lattice of varieties of De Morgan monoids.

Even when we prioritize axiomatic extensions (as relevance logicians have tended to), the completeness conditions in Theorems 4.2 and 6.1(iii),(iii) arise naturally and call for a consideration of subquasivarieties as well. The remainder of this paper therefore attempts to identify the (quasi)varieties of De Morgan monoids that have such properties. We describe completely the varieties that are PSC, and those that have the JEP, and we supply some new information concerning structural completeness. In so doing, we are led to consider two neighbouring families of residuated structures, viz. Dunn monoids and Brouwerian algebras.

Definition 8.1. A De Morgan monoid is an algebra $\boldsymbol{A}=\langle A ; \cdot, \wedge, \vee, \neg, e\rangle$ comprising a distributive lattice $\langle A ; \wedge, \vee\rangle$, a commutative monoid $\langle A ; \cdot, e\rangle$ that is square-increasing (i.e., $\boldsymbol{A}$ satisfies $x \leqslant x^{2}:=x \cdot x$ ), and a function $\neg: A \rightarrow A$, called an involution, such that $\boldsymbol{A}$ satisfies $\neg \neg x \approx x$ and

$$
x \cdot y \leqslant z \Longleftrightarrow x \cdot \neg z \leqslant \neg y .
$$

Here, $\alpha \leqslant \beta$ abbreviates $\alpha \approx \alpha \wedge \beta$. We refer to $\cdot$ as fusion, and we define $f=\neg e$. We denote by DMM the class of all De Morgan monoids (which is a variety, by [22, Thm. 2.7]).

For $\boldsymbol{A}$ as in Definition 8.1, fusion distributes over $\vee$, while $\neg$ is an antiautomorphism of $\langle A ; \wedge, \vee\rangle$, so De Morgan's laws hold. The following facts about any De Morgan monoid $\boldsymbol{A}$ are known (see [48] for sourcing).

(I) $\boldsymbol{A}$ is nontrivial iff its neutral element $e$ is not its least element.

(II) $\boldsymbol{A}$ is simple iff $e$ has just one strict lower bound in $\boldsymbol{A}$.

(III) $\boldsymbol{A}$ is finitely subdirectly irreducible iff $e$ is join-irreducible (or equivalently, join-prime) in $\boldsymbol{A}$.

(IV) $\boldsymbol{A}$ is subdirectly irreducible iff $e$ is completely join-irreducible in $\boldsymbol{A}$.

(V) If $\boldsymbol{A}$ has a least element $\perp$, then $a \cdot \perp=\perp$ for all $a \in A$.

(VI) If $\boldsymbol{A}$ is finitely subdirectly irreducible and $a \in A$, then $e \leqslant a$ or $a \leqslant f$. 
(VII) $\boldsymbol{A}$ satisfies $f \leqslant e$ iff it is idempotent (i.e., $a^{2}=a$ for all $a \in A$ ). In this case $\boldsymbol{A}$ is called a Sugihara monoid. The odd Sugihara monoids are the ones in which $f=e$.

(VIII) $\boldsymbol{A}$ satisfies $x \leqslant f^{2}$ iff it is anti-idempotent, in the sense that the variety $\mathbb{V}(\boldsymbol{A})$ has no nontrivial idempotent member.

(IX) $\boldsymbol{A}$ satisfies $x \leqslant e$ iff it is a Boolean algebra (in which $\cdot$ duplicates $\wedge$ ).

(X) In $\boldsymbol{A}$, we have $f^{3}=f^{2}$.

The variety OSM of all odd Sugihara monoids coincides with $\mathbb{V}(\boldsymbol{S})$ for the algebra $\boldsymbol{S}$ whose universe is the set $\mathbb{Z}$ of all integers, whose lattice order is the usual total order, whose involution $\neg$ is additive inversion, and whose fusion is defined by

$$
a \cdot b=\left\{\begin{array}{l}
\text { the element of }\{a, b\} \text { with greater absolute value, if }|a| \neq|b| \\
a \wedge b \text { if }|a|=|b| .
\end{array}\right.
$$

For each $n \in \omega$, let $\boldsymbol{S}_{2 n+1}$ denote the subalgebra of $\boldsymbol{S}$ with universe

$$
\{-n, \ldots,-1,0,1, \ldots, n\} \text {. }
$$

Up to isomorphism, the algebras $\boldsymbol{S}_{2 n+1}(0<n \in \omega)$ are just the finitely generated subdirectly irreducible odd Sugihara monoids (cf. [1, Sec. 29.4]). Thus, every simple odd Sugihara monoid is isomorphic to $\boldsymbol{S}_{3}$, and the subvariety lattice of OSM is the chain

$$
\mathbb{V}\left(\boldsymbol{S}_{1}\right) \subsetneq \mathbb{V}\left(\boldsymbol{S}_{3}\right) \subsetneq \mathbb{V}\left(\boldsymbol{S}_{5}\right) \subsetneq \ldots \subsetneq \mathbb{V}\left(\boldsymbol{S}_{2 n+1}\right) \subsetneq \ldots \subsetneq \mathbb{V}(\boldsymbol{S})
$$

Theorem 8.2. ([50, 23]) Every quasivariety of odd Sugihara monoids is a variety, i.e., the variety OSM is HSC.

Infinite 1-generated De Morgan monoids exist, but 0-generated De Morgan monoids are finite. Indeed, Slaney [61] proved that the free 0-generated De Morgan monoid has exactly 3088 elements. Its congruence lattice has just 68 elements, no two of which produce isomorphic factor algebras [49, Cor. 3.6]. Let $\boldsymbol{A}_{1}, \ldots, \boldsymbol{A}_{68}$ denote the factor algebras, where $\boldsymbol{A}_{1}$ is trivial. By the Homomorphism Theorem, these are all of the 0-generated De Morgan monoids, up to isomorphism. The minimal quasivarieties of De Morgan monoids are just $\mathbb{V}\left(\boldsymbol{S}_{3}\right)$ and $\mathbb{Q}\left(\boldsymbol{A}_{i}\right), i=2, \ldots, 68$ [49, Thm. 3.4]. As passive structural completeness persists in subquasivarieties, the next result is a characterization of the PSC quasivarieties of De Morgan monoids.

Theorem 8.3. The maximal PSC subquasivarieties of DMM are just the distinct classes $\operatorname{Ret}\left(\mathrm{DMM}, \boldsymbol{A}_{i}\right), i=1, \ldots, 68$, and every nontrivial PSC quasivariety of De Morgan monoids is contained in just one of these.

Moreover, $\operatorname{Ret}\left(\mathrm{DMM}, \boldsymbol{A}_{1}\right)$ is the variety of odd Sugihara monoids. For $i>1$, each relatively simple member of $\operatorname{Ret}\left(\mathrm{DMM}, \boldsymbol{A}_{i}\right)$ is isomorphic to $\boldsymbol{A}_{i}$.

Proof. A De Morgan monoid has a trivial subalgebra iff it is an odd Sugihara monoid, so $\operatorname{Ret}\left(\mathrm{DMM}, \boldsymbol{A}_{1}\right)=\mathrm{OSM}$, and DMM is not a Kollár variety. Therefore, $\operatorname{Ret}\left(\mathrm{DMM}, \boldsymbol{A}_{i}\right)$ is a maximal PSC subquasivariety of DMM, for 
$i=1, \ldots, 68$, by Theorem 7.11(ii), (ii). Every maximal PSC subquasivariety $\mathrm{K}^{\prime}$ of DMM, other than OSM, has a finite nontrivial member (viz. the 0generated subalgebra of any member of $\left.\mathrm{K}^{\prime} \backslash \mathrm{OSM}\right)$, so $\mathrm{K}^{\prime}=\operatorname{Ret}\left(\mathrm{DMM}, \boldsymbol{A}_{i}\right)$ for some $i \in\{2, \ldots, 68\}$, by Theorem 7.11(iii), and every nontrivial PSC subquasivariety of DMM is contained in $\operatorname{Ret}\left(\mathrm{DMM}, \boldsymbol{A}_{i}\right)$ for exactly one $i \in\{1, \ldots, 68\}$, by Theorem 7.11(iv). For $i>1$, the common retract $\boldsymbol{A}_{i}$ of Ret(DMM, $\boldsymbol{A}_{i}$ ) is unique (up to isomorphism) and relatively simple, by Theorem 7.6, since it is 0-generated and nontrivial.

The PSC subvarieties of DMM are more limited. We depict below the two-element Boolean algebra $\mathbf{2}$, the three-element Sugihara monoid $\boldsymbol{S}_{3}$, and two four-element De Morgan monoids, $\boldsymbol{C}_{4}$ and $\boldsymbol{D}_{4}$. In each case, the labeled Hasse diagram determines the structure. Recall that $\mathbb{V}(\mathbf{2})$ is the class of all Boolean algebras.

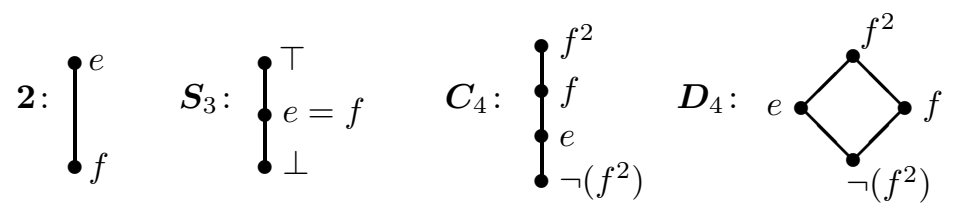

The second item of the next result is due to Slaney [62, Thm.1]. The first item is implicit in [62]; also see [48, Sec. 5].

\section{Theorem 8.4.}

(i) A De Morgan monoid is simple and 0-generated iff it is isomorphic to $\mathbf{2}$ or to $\boldsymbol{C}_{4}$ or to $\boldsymbol{D}_{4}$.

(ii) Let $h: \boldsymbol{A} \rightarrow \boldsymbol{B}$ be a homomorphism, where $\boldsymbol{A}$ is a finitely subdirectly irreducible De Morgan monoid, and $\boldsymbol{B}$ is 0-generated and nontrivial. Then $h$ is an isomorphism or $\boldsymbol{B} \cong \boldsymbol{C}_{4}$.

Every finitely generated subdirectly irreducible Sugihara monoid that is not a Boolean algebra can be mapped homomorphically onto $\boldsymbol{S}_{3}$. (This is well known; see for instance [48, Sec.5].) Consequently, every nontrivial variety of Sugihara monoids contains $\mathbf{2}$ or $\boldsymbol{S}_{3}$. Moreover, a variety of De Morgan monoids consists of Sugihara monoids iff it omits both $\boldsymbol{C}_{4}$ and $\boldsymbol{D}_{4}$ [48, Thm. 5.21], whence

$$
\mathbb{V}(\mathbf{2}), \mathbb{V}\left(\boldsymbol{S}_{3}\right), \mathbb{V}\left(\boldsymbol{C}_{4}\right) \text { and } \mathbb{V}\left(\boldsymbol{D}_{4}\right)
$$

are precisely the minimal varieties of De Morgan monoids [48, Thm.6.1]. (They are distinct, by Jónsson's Theorem.) Theorem 8.4(ii) suggests that $\boldsymbol{C}_{4}$ has more interesting homomorphic pre-images than the other simple 0 -generated De Morgan monoids. We therefore make the abbreviation

$$
\mathrm{N}:=\operatorname{Ret}\left(\mathrm{DMM}, \boldsymbol{C}_{4}\right)=\left\{\boldsymbol{A} \in \mathrm{DMM}:|A|=1 \text { or } \boldsymbol{C}_{4} \text { is a retract of } \boldsymbol{A}\right\} \text {. }
$$

The quasivariety $\mathrm{N}$ is not a variety [49, Sec. 4]. It is therefore not obvious that $\mathrm{N}$ possesses a largest subvariety, but in fact it does. 
Definition 8.5. We denote by $\mathrm{M}$ the variety of De Morgan monoids satisfying $e \leqslant f$ and $x \leqslant f^{2}$ and

$$
f^{2} \cdot \neg((f \cdot x) \wedge(f \cdot \neg x)) \approx f^{2} .
$$

Theorem 8.6. ([49, Thms. 4.13, 6.8]) $\mathrm{M}$ is the largest subvariety of $\mathrm{N}$, and $\mathrm{M}$ has $2^{\aleph_{0}}$ distinct subvarieties.

We can now isolate the PSC varieties of De Morgan monoids.

Theorem 8.7. Let $\mathrm{K}$ be a variety of De Morgan monoids. Then $\mathrm{K}$ is PSC iff one of the following four (mutually exclusive) conditions holds:

(i) $\mathrm{K}$ is the variety $\mathbb{V}(\mathbf{2})$ of all Boolean algebras;

(ii) $\mathrm{K}=\mathbb{V}\left(\boldsymbol{D}_{4}\right)$;

(iii) K consists of odd Sugihara monoids;

(iv) $\mathrm{K}$ is a nontrivial subvariety of $\mathrm{M}$.

Proof. By Theorem 8.3, a nontrivial variety of De Morgan monoids is PSC iff it lies within $\operatorname{Ret}\left(\mathrm{DMM}, \boldsymbol{A}_{i}\right)$ for some $i \in\{1, \ldots, 68\}$ (in which case $i$ is unique). This includes all the varieties mentioned in the present theorem, because $\mathbf{2}, \boldsymbol{C}_{4}, \boldsymbol{D}_{4}$ and the trivial De Morgan monoid are 0-generated and finite. Conversely, consider a nontrivial PSC variety $\mathrm{K} \subseteq \operatorname{Ret}\left(\mathrm{DMM}, \boldsymbol{A}_{i}\right)$. As $\operatorname{Ret}\left(\mathrm{DMM}, \boldsymbol{A}_{1}\right)=\mathrm{OSM}$, we may assume that $i>1$. Theorem 8.3 also asserts that $\boldsymbol{A}_{i}$ is relatively simple in the quasivariety $\operatorname{Ret}\left(\mathrm{DMM}, \boldsymbol{A}_{i}\right)$, so it is a simple member of K. Therefore, $\boldsymbol{A}_{i} \in \mathbb{I}\left(\mathbf{2}, \boldsymbol{C}_{4}, \boldsymbol{D}_{4}\right)$, by Theorem 8.4(i). If $\boldsymbol{A}_{i} \cong \boldsymbol{C}_{4}$ then $\mathrm{K} \subseteq \mathrm{M}$, by Theorem [8.6. so suppose $\boldsymbol{A}_{i} \cong \mathbf{2}$ [resp. $\boldsymbol{A}_{i} \cong \boldsymbol{D}_{4}$ ]. Let $\boldsymbol{B} \in \mathrm{K}$ be subdirectly irreducible. As $\boldsymbol{A}_{i} \in \mathbb{H}(\boldsymbol{B})$, Theorem 8.4(ii) shows that $\boldsymbol{B} \cong \boldsymbol{A}_{i}$. Consequently, $\mathrm{K}$ is $\mathbb{V}(\mathbf{2})$ [resp. $\mathbb{V}\left(\boldsymbol{D}_{4}\right)$ ].

Because the four minimal varieties of De Morgan monoids are locally finite and congruence distributive, they are minimal as quasivarieties, by Theorem 7.12, In particular, $\mathbb{V}(\mathbf{2})$ and $\mathbb{V}\left(\boldsymbol{D}_{4}\right)$ are HSC, and so is OSM (as we saw in Theorem 8.2).

By Theorem 8.7, every remaining SC variety of De Morgan monoids must be a subvariety of $\mathrm{M}$. By Theorem 9.6 below, $\mathrm{M}$ and the quasivariety $\mathrm{N}$ are not HSC. (We conjecture that $\mathrm{M}$ and $\mathrm{N}$ are not SC.) In the lattice of subvarieties of $\mathrm{M}$, the unique atom $\mathbb{V}\left(\boldsymbol{C}_{4}\right)$ has just six covers, identified in [49, Thm. 8.10]. The varietal join of those six covers is HSC [49, Thm. 8.13].

The next result characterizes the JEP for subvarieties of DMM.

Theorem 8.8. Let $\mathrm{K}$ be a variety of De Morgan monoids. Then $\mathrm{K}$ has the JEP iff one of the following (mutually exclusive) conditions is met.

(i) $\mathrm{K}$ is $P S C$.

(ii) $\mathrm{K}=\mathbb{V}(\boldsymbol{A})$ for some simple De Morgan monoid $\boldsymbol{A}$ such that $\boldsymbol{D}_{4}$ is a proper subalgebra of $\boldsymbol{A}$.

(iii) There exist $\boldsymbol{A}, \boldsymbol{B}$ such that $\mathrm{K}=\mathbb{Q}(\boldsymbol{B}), \boldsymbol{A}$ is a simple subalgebra of $\boldsymbol{B}$, and $\boldsymbol{C}_{4}$ is a proper subalgebra of $\boldsymbol{A}$. 
In (iii), ' $\mathrm{K}=\mathbb{Q}(\boldsymbol{B})$ ' can be paraphrased as ' $\mathrm{K}=\mathbb{V}(\boldsymbol{B})$ and every finitely generated subdirectly irreducible member of $\mathbb{H}_{\mathrm{U}}(\boldsymbol{B})$ belongs to $\mathbb{I S P}_{\mathrm{U}}(\boldsymbol{B})$ '.

Proof. Sufficiency follows from Theorems 4.2, 4.3 and 5.10, since DMM has EDPC (by [22, Thm. 3.55]).

Conversely, suppose that $\mathrm{K}$ has the JEP but is not PSC. Then $\mathrm{K}$ is nontrivial and, by Theorem 8.7. $\mathrm{K}$ does not consist solely of Boolean algebras, nor solely of odd Sugihara monoids. In particular, not every member of $\mathrm{K}$ has a trivial subalgebra. Therefore, $\mathrm{K}$ is a Kollár variety, by Proposition 5.5(iii), so $\boldsymbol{S}_{3} \notin \mathrm{K}$. As every finitely generated subdirectly irreducible Sugihara monoid that is not a Boolean algebra maps homomorphically onto $\boldsymbol{S}_{3}$, no such algebra belongs to $\mathrm{K}$, whence every idempotent member of $\mathrm{K}$ is Boolean. Consequently, if $\mathrm{K}$ has an idempotent member, then the 0generated subalgebras of its nontrivial members are all isomorphic to $\mathbf{2}$, by Proposition 5.5(i). In that case, $\mathrm{K}$ consists of idempotent algebras, by (VII), and so coincides with $\mathbb{V}(\mathbf{2})$, a contradiction. This shows that $\mathrm{K}$ has no nontrivial idempotent member.

Being nontrivial, $\mathrm{K}$ therefore includes $\boldsymbol{C}_{4}$ or $\boldsymbol{D}_{4}$, so $\mathbb{I}\left(\boldsymbol{C}_{4}\right)$ or $\mathbb{I}\left(\boldsymbol{D}_{4}\right)$ is the class of all 0-generated nontrivial members of K, by Proposition 5.5(1). Also, by (VIII), $\mathrm{K}$ satisfies $x \leqslant f^{2}$ (and hence $\neg\left(f^{2}\right) \leqslant x$ as well). On the other hand, $\mathrm{K} \nsubseteq \mathrm{M}$ and $\mathrm{K} \neq \mathbb{V}\left(\boldsymbol{D}_{4}\right)$, by Theorem 8.7, as $\mathrm{K}$ is not PSC.

By Theorem 5.6, there is a simple De Morgan monoid $\boldsymbol{A} \in \mathrm{K}$ such that

$$
\text { every simple member of } \mathrm{K} \text { belongs to } \mathbb{I S P}_{U}(\boldsymbol{A}) \text {. }
$$

By Theorem 4.2, there exists $\boldsymbol{E} \in \mathrm{K}$ such that $\mathrm{K}=\mathbb{Q}(\boldsymbol{E})=\mathbb{I P}_{\mathrm{S}} \mathbb{S P}_{\mathrm{U}}(\boldsymbol{E})$, whence $\boldsymbol{A} \in \mathbb{I S P}_{\mathrm{U}}(\boldsymbol{E})$ (as $\boldsymbol{A}$ is simple). Choose $\boldsymbol{B} \in \mathbb{P P}_{\mathrm{U}}(\boldsymbol{E})$ with $\boldsymbol{A} \in \mathbb{S}(\boldsymbol{B})$. As $\boldsymbol{E} \in \mathbb{I} \mathbb{S}(\boldsymbol{B})$, we have $\mathrm{K}=\mathbb{Q}(\boldsymbol{B})$.

Suppose first that $\mathbb{I}\left(\boldsymbol{C}_{4}\right)$ is the class of 0-generated nontrivial members of K. As $\mathrm{K}$ is a Kollár variety, the 0-generated subalgebra of $\boldsymbol{A}$ is nontrivial, so it can be identified with $\boldsymbol{C}_{4}$. If $\boldsymbol{A}=\boldsymbol{C}_{4}$, then every simple member of K is isomorphic to $\boldsymbol{C}_{4}$, by (5), so $\boldsymbol{C}_{4}$ is a retract of every nontrivial member of $\mathrm{K}$ (by Corollary 5.4 and Remark 7.10), i.e., $\mathrm{K} \subseteq \mathrm{M}$, a contradiction. This shows that $\boldsymbol{C}_{4}$ is a proper subalgebra of $\boldsymbol{A}$, so (iii) holds.

We may now assume that $\mathbb{I}\left(\boldsymbol{D}_{4}\right)$ is the class of 0 -generated nontrivial members of K. Let $\boldsymbol{G}$ be any subdirectly irreducible member of K. Again, since $\mathrm{K}$ is a Kollár variety, the 0-generated subalgebras of $\boldsymbol{A}, \boldsymbol{G}$ are nontrivial, so we may assume that $\boldsymbol{D}_{4} \in \mathbb{S}(\boldsymbol{A}) \cap \mathbb{S}(\boldsymbol{G})$. Therefore, as $\boldsymbol{D}_{4}$ satisfies $e \wedge f \approx \neg\left(f^{2}\right)$, so does $\boldsymbol{G}$. Consequently, as $\neg\left(f^{2}\right)$ is the least element of $\boldsymbol{G}$, it follows from (VI) that $\neg\left(f^{2}\right)$ is the greatest strict lower bound of $e$ in $\boldsymbol{G}$, whence $\boldsymbol{G}$ is simple. This shows that $\mathrm{K}$ is a semisimple variety, so $\mathrm{K}=\mathbb{Q}(\boldsymbol{A})$, by (5) . Since $\mathrm{K} \neq \mathbb{V}\left(\boldsymbol{D}_{4}\right)=\mathbb{Q}\left(\boldsymbol{D}_{4}\right)$, we must have $\boldsymbol{A} \neq \boldsymbol{D}_{4}$, and so (ii) holds.

Note that (ii) precludes both (iii) and (iii), by Theorem 7.6, because each of $\boldsymbol{C}_{4}, \boldsymbol{D}_{4}$ has no retract other than its isomorphic images, and cannot be 
a retract of a strictly larger simple algebra. Also, (iii) precludes (iii), by Proposition 5.5(i), as $\boldsymbol{C}_{4}$ and $\boldsymbol{D}_{4}$ are both 0-generated and nontrivial.

As every variety is generated as such by its finitely generated subdirectly irreducible members, the paraphrase in the last claim is justified by Jónsson's Theorem and the CEP for De Morgan monoids (which implies that $\mathbb{H} \mathbb{S}(\boldsymbol{P}) \subseteq \mathbb{S H}(\boldsymbol{P})$ for all $\boldsymbol{P} \in \mathrm{DMM})$.

Corollary 8.9. A variety of Sugihara monoids has the JEP iff it is PSC.

Corollary 8.10. In the lattice of varieties of De Morgan monoids, all but four of the join-irreducible covers of atoms have the JEP.

Proof. The join-irreducible covers of the four atoms are described in [49, Thm. 7.2]. With four exceptions, each has the JEP, as it is either a subvariety of M or of OSM (and is thus PSC) or has the form $\mathbb{V}(\boldsymbol{A})$ for a simple algebra $\boldsymbol{A}$. The exceptions are covers of $\mathbb{V}\left(\boldsymbol{C}_{4}\right)$ that lack the JEP, by Proposition 5.5(i), because they are the varietal closures of 0-generated algebras $\boldsymbol{C}_{5}, \ldots, \boldsymbol{C}_{8}$ (respectively), each of which has more elements than $\boldsymbol{C}_{4}$.

In Theorem 8.8(iii), it can happen that $\mathrm{K}$ is not generated, even as a variety, by one finitely subdirectly irreducible algebra. This remains the case when $\mathrm{K}=\mathbb{Q}(\boldsymbol{B})$ for some finite $\boldsymbol{B}$. These claims will be justified in Example 9.7

Example 8.11. A relevant algebra is an $e$-free subreduct of a De Morgan monoid (i.e., a subalgebra of the reduct $\langle A ; \cdot, \wedge, \vee, \neg\rangle$ of some $\boldsymbol{A} \in \mathrm{DMM}$ ). These algebras form a variety $R A$, algebraizing the fragment $\mathbf{R}$ of $\mathbf{R}^{\mathbf{t}}$ that lacks the so-called Ackermann constants. A finite equational basis for RA is given in [21] (also see [18, [26, Cor. 4.11] and [48, Sec. 7]). Boolean algebras may be regarded as relevant algebras, since they satisfy $e \approx x \vee \neg x$.

The variety RA respects the relevance principle of Definition 5.11 , by 38 , Thm.6] (since every finite set of equations in its signature is consistent over $\mathrm{RA}$, as follows from a consideration of the locally finite $e$-free reduct of the odd Sugihara monoid $\boldsymbol{S}$ ). Therefore, RA has the JEP, by Theorem 5.12 , In other words (by Theorem 4.2), RA $=\mathbb{Q}(\boldsymbol{A})$ for some $\boldsymbol{A}$ (cf. [65, Thm. 5]). This $\boldsymbol{A}$ is not $\boldsymbol{F}_{\mathrm{RA}}\left(\aleph_{0}\right)$, as RA is not (even passively) structurally complete. In fact, RA has no nontrivial PSC subvariety, other than $\mathbb{V}(2)$ [58, Thm.6].

This contrasts with the fact that DMM lacks the JEP (by Proposition 5.5(i), as it has non-isomorphic 0-generated nontrivial members). Because $\mathbf{2}$ has no trivial subalgebra, while the $e$-free reduct of $\boldsymbol{S}_{3}$ has a trivial subalgebra (and so belongs to no Kollár quasivariety), RA would violate Proposition [5.5(iii) if we dropped the demand there for a constant symbol in the signature. $\square$

We have seen that M is PSC and contains all the SC subvarieties of DMM not explicitly identified in Theorem 8.7. We shall show, however, that $M$ has $2^{\aleph_{0}}$ structurally incomplete subvarieties. For this we need to consider algebras called Dunn monoids, and a construction known as reflection. 


\section{Dunn Monoids And Reflections}

With respect to the derived operation $x \rightarrow y:=\neg(x \cdot \neg y)$, every De Morgan monoid satisfies $\neg x \approx x \rightarrow f$ and

$$
x \cdot y \leqslant z \Longleftrightarrow y \leqslant x \rightarrow z \quad \text { (the law of residuation). }
$$

Definition 9.1. An algebra $\boldsymbol{A}=\langle A ; \cdot, \rightarrow, \wedge, \vee, e\rangle$ is called a Dunn monoid if $\langle A ; \wedge, \vee\rangle$ is a distributive lattice, $\langle A ; \cdot, e\rangle$ is a square-increasing commutative monoid and $\rightarrow$ is a binary operation-called residuation - such that $\boldsymbol{A}$ satisfies (6).

Dunn monoids form a variety, again by [22, Thm. 2.7]. This variety is PSC, because $\{e\}$ constitutes a subalgebra of any Dunn monoid. Clearly, up to term equivalence, every De Morgan monoid has a reduct that is a Dunn monoid. Conversely, as recounted below, each Dunn monoid can be embedded into (the $\cdot, \rightarrow, \wedge, \vee$, e reduct of) a De Morgan monoid. Properties (II) -(V) of De Morgan monoids remain true for Dunn monoids.

Definition 9.2. (cf. Meyer [44]) Given a Dunn monoid $\boldsymbol{A}$ and a disjoint copy $A^{\prime}=\left\{a^{\prime}: a \in A\right\}$ of $A$, let $\perp, \top$ be distinct non-elements of $A \cup A^{\prime}$. By the reflection $\mathrm{R}(\boldsymbol{A})$ of $\boldsymbol{A}$, we mean the De Morgan monoid with universe $\mathrm{R}(A)=A \cup A^{\prime} \cup\{\perp, \top\}$ such that $\boldsymbol{A}$ is a subalgebra of the Dunn monoid reduct of $\mathrm{R}(\boldsymbol{A})$ and, for all $a, b \in A$ and $x \in \mathrm{R}(A)$,

$$
\begin{aligned}
& x \cdot \perp=\perp<a<b^{\prime}<\top=a^{\prime} \cdot b^{\prime}, \text { and if } x \neq \perp, \text { then } x \cdot \top=\top ; \\
& a \cdot b^{\prime}=(a \rightarrow b)^{\prime} ; \\
& \neg a=a^{\prime} \text { and } \neg\left(a^{\prime}\right)=a \text { and } \neg \perp=\top \text { and } \neg \top=\perp .
\end{aligned}
$$

In Definition [9.2, since $f=e^{\prime}$, we have $\top=f^{2}$ and $\perp=\neg\left(f^{2}\right)$, so $\perp, \top$ belong to every subalgebra of $\mathrm{R}(\boldsymbol{A})$. The subalgebra on $\left\{\perp, e, e^{\prime}, \top\right\}$ is isomorphic to $\boldsymbol{C}_{4}$. The De Morgan monoid $\mathrm{R}(\boldsymbol{A})$ satisfies $e \leqslant f$ and $x \leqslant f^{2}$ and (4), so it belongs to $\mathrm{M}$. The construction of $\mathrm{R}(\boldsymbol{A})$ from $\boldsymbol{A}$ preserves and reflects [finite] subdirect irreducibility - except that $\mathrm{R}(\boldsymbol{A})$ is subdirectly irreducible when $\boldsymbol{A}$ is trivial. The interaction between reflections and the operators $\mathbb{S}, \mathbb{H}, \mathbb{P}_{\mathrm{U}}$ is illuminated in the next lemma.

Lemma 9.3. ([49, Lem. 6.5]) Let $\boldsymbol{A}$ be a Dunn monoid.

(i) If $\boldsymbol{B}$ is a subalgebra of $\boldsymbol{A}$, then $B \cup\left\{b^{\prime}: b \in B\right\} \cup\{\perp, \top\}$ is the universe of a subalgebra of $\mathrm{R}(\boldsymbol{A})$ that is isomorphic to $\mathrm{R}(\boldsymbol{B})$, and every subalgebra of $\mathrm{R}(\boldsymbol{A})$ arises in this way from a subalgebra of $\boldsymbol{A}$.

(ii) If $\theta$ is a congruence of $\boldsymbol{A}$, then

$$
\mathrm{R}(\theta):=\theta \cup\left\{\left\langle a^{\prime}, b^{\prime}\right\rangle:\langle a, b\rangle \in \theta\right\} \cup\{\langle\perp, \perp\rangle,\langle\top, \top\rangle\}
$$

is a congruence of $\mathrm{R}(\boldsymbol{A})$, and $\mathrm{R}(\boldsymbol{A}) / \mathrm{R}(\theta) \cong \mathrm{R}(\boldsymbol{A} / \theta)$. Also, every proper congruence of $\mathrm{R}(\boldsymbol{A})$ has the form $\mathrm{R}(\theta)$ for some $\theta \in$ Con $\boldsymbol{A}$.

(iii) If $\left\{\boldsymbol{A}_{i}: i \in I\right\}$ is a family of Dunn monoids and $\mathcal{U}$ is an ultrafilter over $I$, then $\prod_{i \in I} \mathrm{R}\left(\boldsymbol{A}_{i}\right) / \mathcal{U} \cong \mathrm{R}\left(\prod_{i \in I} \boldsymbol{A}_{i} / \mathcal{U}\right)$. 
Definition 9.4. The reflection of a variety $\mathrm{K}$ of Dunn monoids is the subvariety $\mathbb{R}(\mathrm{K}):=\mathbb{V}\{\mathrm{R}(\boldsymbol{A}): \boldsymbol{A} \in \mathrm{K}\}$ of $\mathrm{M}$.

As a function from the lattice of varieties of Dunn monoids to the subvariety lattice of $M$, the operator $\mathbb{R}$ is obviously isotone. It is also orderreflecting, and therefore injective [49, Lem. 6.7].

Theorem 9.5. Let $\mathrm{K}$ be a variety of Dunn monoids. If $\mathbb{R}(\mathrm{K})$ is structurally complete, then so is $\mathrm{K}$ (i.e., $\mathbb{R}$ preserves structural incompleteness).

Proof. Suppose $\mathrm{K}$ is not $\mathrm{SC}$, so $\mathrm{K}=\mathbb{H}(\mathrm{L})$ for some quasivariety $\mathrm{L} \subsetneq \mathrm{K}$. Now $\mathrm{L}^{\dagger}:=\mathbb{I}\{\mathrm{R}(\boldsymbol{B}): \boldsymbol{B} \in \mathrm{L}\}$ is closed under $\mathbb{S}$ and $\mathbb{P}_{\mathbb{U}}$, by Lemma 9.3((ii),(iii), so $\mathbb{Q}\left(\mathrm{L}^{\dagger}\right)=\mathbb{I P}_{\mathbb{S}}\left(\mathrm{L}^{\dagger}\right) \subseteq \mathbb{R}(\mathrm{K})$. As $\mathrm{L} \subsetneq \mathrm{K}$, and because all quasivarieties are closed under subdirect products, there is an algebra $\boldsymbol{A} \in \mathrm{K}_{S I} \backslash \mathrm{L}$. Then $\mathrm{R}(\boldsymbol{A})$ belongs to $\mathbb{R}(\mathrm{K})$ and is subdirectly irreducible. So, if $\mathrm{R}(\boldsymbol{A}) \in \mathbb{Q}\left(\mathrm{L}^{\dagger}\right)$, then $\mathrm{R}(\boldsymbol{A}) \cong \mathrm{R}(\boldsymbol{B})$ for some $\boldsymbol{B} \in \mathrm{L}$, whence $\boldsymbol{A} \cong \boldsymbol{B}$, contradicting the fact that $\boldsymbol{A} \notin \mathrm{L}$. Therefore, $\mathrm{R}(\boldsymbol{A}) \notin \mathbb{Q}\left(\mathrm{L}^{\dagger}\right)$, and so $\mathbb{Q}\left(\mathrm{L}^{\dagger}\right) \neq \mathbb{R}(\mathrm{K})$.

We claim that $\mathbb{R}(\mathrm{K})=\mathbb{V}\left(\mathrm{L}^{\dagger}\right)$. To see this, let $\boldsymbol{C} \in \mathbb{R}(\mathrm{K})_{S I}$. By Jónsson's Theorem and Lemma 9.3, $\boldsymbol{C} \cong \mathrm{R}(\boldsymbol{D})$ for some $\boldsymbol{D} \in \mathrm{K}$. As $\mathrm{K}=\mathbb{H}(\mathrm{L})$, we may assume that $\boldsymbol{D}=\boldsymbol{E} / \theta$ for some $\boldsymbol{E} \in \mathrm{L}$ and some $\theta \in$ Con $\boldsymbol{E}$. Then $\boldsymbol{C} \cong \mathrm{R}(\boldsymbol{E} / \theta) \cong \mathrm{R}(\boldsymbol{E}) / \mathrm{R}(\theta)$, by Lemma 9.3(iii), whence $\boldsymbol{C} \in \mathbb{H}\left(\mathrm{L}^{\dagger}\right) \subseteq \mathbb{V}\left(\mathrm{L}^{\dagger}\right)$. This vindicates the claim.

In summary, $\mathbb{Q}\left(\mathrm{L}^{\dagger}\right)$ is a proper subquasivariety of $\mathbb{R}(\mathrm{K})$ that fails to generate a proper subvariety of $\mathbb{R}(\mathrm{K})$, so $\mathbb{R}(\mathrm{K})$ is not $\mathrm{SC}$.

A Dunn (or De Morgan) monoid is said to be semilinear if it is isomorphic to a subdirect product of totally ordered algebras. The semilinear Dunn monoids form a structurally incomplete variety [51, Thm. 9.4]. Its reflection is just the variety SLM of semilinear members of $\mathrm{M}$, by Lemma 9.3 and [49, Cor. 5.8], so SLM is not structurally complete either, by Theorem 9.5, This confirms that $\mathrm{M}$ is not HSC (and likewise $\mathrm{N}$ ), but we can say more:

Theorem 9.6. The variety $\mathrm{M}$ has $2^{\aleph_{0}}$ structurally incomplete subvarieties.

Proof. Since the operator $\mathbb{R}$ is injective, it suffices, by Theorem 9.5, to exhibit $2^{\aleph_{0}}$ structurally incomplete varieties of Dunn monoids. The existence of such a family will be proved in Theorem 10.8 below.

We conclude this section with an example illustrating Theorem 8.8(iii).

Example 9.7. The Dunn monoid reduct of a De Morgan monoid $\boldsymbol{A}$ shall be denoted by $\boldsymbol{A}^{+}$. We then denote by $X(\boldsymbol{A})$ the De Morgan monoid that extends $\mathrm{R}\left(\boldsymbol{A}^{+}\right)$by just one element $x$, where $a<x<b^{\prime}$ for all $a, b \in A$, and $x \cdot \neg\left(f^{2}\right)=\neg\left(f^{2}\right)$ and $x=\neg x=x \cdot c$ and $x \cdot d=f^{2}$ whenever $\neg\left(f^{2}\right)<c \leqslant x<d \leqslant f^{2}$. (It is easily checked that this $X(\boldsymbol{A})$ is indeed a De Morgan monoid, with $\mathrm{R}\left(\boldsymbol{A}^{+}\right) \in \mathbb{S}(X(\boldsymbol{A}))$.) 
Let $\mathrm{K}=\mathbb{V}\left(X\left(\mathbf{2} \times \boldsymbol{S}_{3}\right)\right)$. As $\mathrm{K}$ is generated by one finite algebra, its finitely subdirectly irreducible members are finite and can be computed mechanically, by Jónsson's Theorem. None of them has the property that its $\mathbb{H} \mathbb{S}$-closure contains all the others, but all of them embed into $X\left(\mathbf{2} \times \boldsymbol{S}_{3}\right)$ (excepting the trivial algebra). Therefore, $\mathrm{K}$ is not generated as a variety by a single (finitely) subdirectly irreducible algebra, but $\mathrm{K}=\mathbb{Q}\left(X\left(\mathbf{2} \times \boldsymbol{S}_{3}\right)\right)$, so $\mathrm{K}$ has the JEP, by Theorem 4.2. For the trivial De Morgan monoid $\boldsymbol{E}$, the five-element simple algebra $X(\boldsymbol{E})$ belongs to $\mathrm{K}$ and has $\boldsymbol{C}_{4}$ as its smallest subalgebra. As cases (ii) and (iii) of Theorem 8.8 are mutually exclusive, $\mathrm{K}$ is not PSC (in contrast with Example 5.9).

\section{Brouwerian Algebras}

Definition 10.1. A Dunn monoid is called a Brouwerian algebra if it satisfies $x \cdot y \approx x \wedge y$ (or equivalently, $x \leqslant e$ ), in which case it is identified with its $\rightarrow, \wedge, \vee, e$ reduct.

A Heyting algebra is therefore just a Brouwerian algebra with a distinguished least element. A well-known duality exists between Heyting algebras and 'Esakia spaces' [19, 20]. It entails a duality between Brouwerian algebras and 'pointed Esakia spaces' (see [5, Sec.3], for instance). Here we require only a topology-free version of the latter duality, explained briefly below.

In an indicated partially ordered set ('poset') $\boldsymbol{X}=\langle X ; \leqslant\rangle$, we define

$$
\uparrow x=\{y \in X: x \leqslant y\} \text { and } \uparrow U=\bigcup_{u \in U} \uparrow u,
$$

for $U \cup\{x\} \subseteq X$, and if $U=\uparrow U$, we call $U$ an up-set of $\boldsymbol{X}$. We define $\downarrow x$ and $\downarrow U$ dually. (Where ambiguity is a danger, we write $\uparrow x$ as $\uparrow^{\boldsymbol{X}} x$, etc.) We call $\boldsymbol{X}$ a dominated [resp. bounded] poset if it has a greatest [resp. a greatest and a least] element. When $\boldsymbol{X}$ is dominated, the set $U p(\boldsymbol{X})$ of all non-empty up-sets of $\boldsymbol{X}$ is closed under intersections and if, for $U, V \in U p(\boldsymbol{X})$, we define

$$
U \rightarrow V:=X \backslash \downarrow(U \backslash V) \quad(=\bigcup\{W \in U p(\boldsymbol{X}): W \cap U \subseteq V\})
$$

then $\boldsymbol{X}^{*}:=\langle U p(\boldsymbol{X}) ; \rightarrow, \cap, \cup, X\rangle$ is a Brouwerian algebra. Note that $\boldsymbol{X}^{*}$ is subdirectly irreducible iff $\boldsymbol{X}$ is bounded and not a singleton.

A function $g: \boldsymbol{X} \rightarrow \boldsymbol{Y}$ between dominated posets is called a $p$-morphism if it is isotone and

$$
\text { whenever } g(x) \leqslant y \in Y \text {, then } y=g(z) \text { for some } z \in \uparrow x \text {. }
$$

In this case, $\uparrow g(x)=g[\uparrow x]$ for all $x \in X$ (whence $g[X] \in U p(\boldsymbol{Y})$ ), $g$ preserves top elements, and there is a homomorphism $g^{*}: \boldsymbol{Y}^{*} \rightarrow \boldsymbol{X}^{*}$, defined by $V \mapsto g^{-1}[V]:=\{x \in X: g(x) \in V\}(V \in U p(\boldsymbol{Y}))$. In particular, each $U \in U p(\boldsymbol{X})$ is the universe of a dominated subposet $\boldsymbol{U}$ of $\boldsymbol{X}$, and since the inclusion map $i: \boldsymbol{U} \rightarrow \boldsymbol{X}$ is a $p$-morphism, the function $i^{*}: V \mapsto U \cap V$ $(V \in U p(\boldsymbol{X}))$ is a homomorphism $\boldsymbol{X}^{*} \rightarrow \boldsymbol{U}^{*}$. As it is obviously surjective, $\boldsymbol{U}^{*} \in \mathbb{H}\left(\boldsymbol{X}^{*}\right)$ for all $U \in U p(\boldsymbol{X})$. 
For a Brouwerian algebra $\boldsymbol{A}$, we denote by $\operatorname{Pr}(\boldsymbol{A})$ the set of all prime filters of the lattice $\langle A ; \wedge, \vee\rangle$, including $A$ itself. Thus, $\operatorname{Pr}(\boldsymbol{A})$ consists of the non-empty up-sets $P$ of the poset reduct $\langle A ; \leqslant\rangle$ of $\boldsymbol{A}$ such that $P$ is closed under $\wedge$ and $A \backslash P$ is closed under $\vee$. The dominated poset $\langle\operatorname{Pr}(\boldsymbol{A}) ; \subseteq\rangle$ is abbreviated as $\boldsymbol{A}_{*}$. It is bounded iff $\boldsymbol{A}$ is finitely subdirectly irreducible. If $h: \boldsymbol{A} \rightarrow \boldsymbol{B}$ is a homomorphism between Brouwerian algebras, then there is a $p$-morphism $h_{*}: \boldsymbol{B}_{*} \rightarrow \boldsymbol{A}_{*}$, defined by $Q \mapsto h^{-1}[Q](Q \in \operatorname{Pr}(\boldsymbol{B}))$.

For each Brouwerian algebra $\boldsymbol{A}$, there is an embedding $\boldsymbol{A} \rightarrow \boldsymbol{A}_{*}{ }^{*}$, defined by $a \mapsto\{P \in \operatorname{Pr}(\boldsymbol{A}): a \in P\}$; it is an isomorphism if $\boldsymbol{A}$ is finite. For each dominated poset $\boldsymbol{X}$, there is an injective $p$-morphism $\boldsymbol{X} \rightarrow \boldsymbol{X}^{*}{ }^{*}$, defined by $x \mapsto\{U \in U p(\boldsymbol{X}): x \in U\}$; it is bijective when $\boldsymbol{X}$ is finite, in which case its inverse is also a $p$-morphism.

The functor $\boldsymbol{A} \mapsto \boldsymbol{A}_{*} ; h \mapsto h_{*}$ defines a duality from the category FBA of finite Brouwerian algebras (and their homomorphisms) to the category FDP of finite dominated posets (and their $p$-morphisms), i.e., it defines a category equivalence from FBA to the opposite category of FDP. A reverse functor is given by $\boldsymbol{X} \mapsto \boldsymbol{X}^{*} ; g \mapsto g^{*}$.

In particular, every finite dominated poset is isomorphic to $\boldsymbol{A}_{*}$ for some finite Brouwerian algebra $\boldsymbol{A}$, and if $\boldsymbol{A}, \boldsymbol{B}$ are finite Brouwerian algebras, then the rule $h \mapsto h_{*}$ defines a bijection from the set of all homomorphisms $\boldsymbol{A} \rightarrow \boldsymbol{B}$ to the set of all $p$-morphisms $\boldsymbol{B}_{*} \rightarrow \boldsymbol{A}_{*}$.

One feature of the above duality is the following (cf. [5, Lem. 3.4(ii)]).

Lemma 10.2. A homomorphism $h$ between finite Brouwerian algebras is surjective iff $h_{*}$ is injective. Also, $h$ is injective iff $h_{*}$ is surjective.

Consequently, a p-morphism $g$ between finite dominated posets is surjective [resp. injective] iff $g^{*}$ is injective [resp. surjective].

Definition 10.3. In a dominated poset $\boldsymbol{X}$, the depth of an element $x$ is the largest non-negative integer $n$ (if it exists) such that the subposet $\uparrow x$ contains a chain of cardinality $n+1$. Thus, the greatest element of $\boldsymbol{X}$ has depth 0 . If $n$ is minimal such that all elements of $\boldsymbol{X}$ have depth at most $n$, then $\boldsymbol{X}$ itself is said to have depth $n$. A variety $\mathrm{K}$ of Brouwerian algebras is said to have depth $n$ if $\boldsymbol{A}_{*}$ has depth at most $n$ for every $\boldsymbol{A} \in \mathrm{K}$.

By (7), for any $p$-morphism $g: \boldsymbol{X} \rightarrow \boldsymbol{Y}$ between dominated posets, if $x \in X$ has depth $n \in \omega$, then $g(x)$ has depth at most $n$.

Each dominated poset $\boldsymbol{X}$ is an up-set of a dominated poset $\widehat{\boldsymbol{X}}$, which differs from $\boldsymbol{X}$ only as follows: whenever $a, b$ are distinct elements of depth 2 in $\boldsymbol{X}$, then $\widehat{\boldsymbol{X}}$ has a (new) element $e_{a b}$ that has no strict lower bound; the strict upper bounds of $e_{a b}$ are just the elements of $\uparrow^{\boldsymbol{X}}\{a, b\}$. (Note: $e_{a b}$ and $e_{b a}$ are the same element.)

Observe that if $\boldsymbol{X}$ has depth $n$, then so does $\widehat{\boldsymbol{X}}$, unless $n=2$ (in which case $\widehat{\boldsymbol{X}}$ has depth 3$)$. Also, since $X \in U p(\widehat{\boldsymbol{X}})$, we always have $\boldsymbol{X}^{*} \in \mathbb{H}\left(\widehat{\boldsymbol{X}}^{*}\right)$. 
The hat construction is illustrated below for a poset $\boldsymbol{P}_{6}$ that will play a role in subsequent arguments.
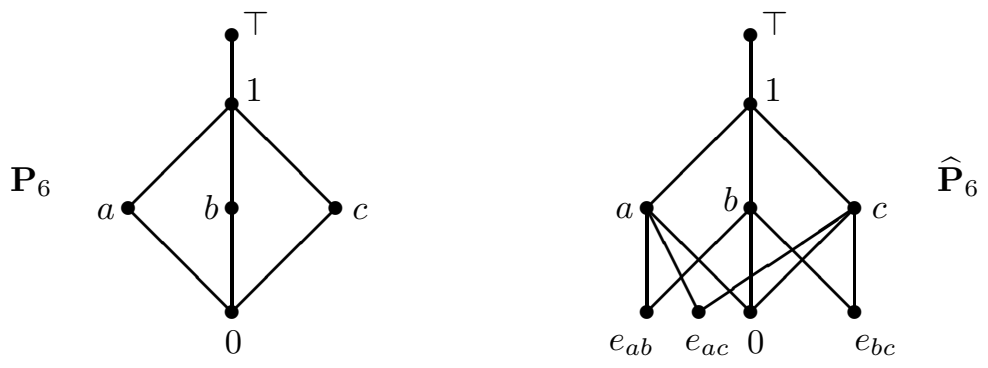

For each positive integer $n$, in the $n$-th direct power of the two-element chain, let $\boldsymbol{K}_{n}$ be the subposet consisting of the least element, the greatest element, the $n$ atoms and the $n$ co-atoms. Note that $\boldsymbol{K}_{n}$ is bounded and has depth 3. Each atom of $\boldsymbol{K}_{n}$ is dominated by just $n-1$ co-atoms, and each co-atom dominates just $n-1$ atoms. Let

$$
\mathcal{K}:=\text { the power set of }\left\{\boldsymbol{K}_{n}: 3 \leq n \in \mathbb{N}\right\},
$$

so $|\mathcal{K}|=2^{\aleph_{0}}$. Kuznetsov 34 proved that there are $2^{\aleph_{0}}$ distinct varieties of Brouwerian algebras of depth 3 , by establishing the following:

$$
\text { for any } C, D \in \mathcal{K} \text {, if } C \neq D \text {, then } \mathbb{V}\left(C^{*}\right) \neq \mathbb{V}\left(D^{*}\right) \text {, }
$$

where $C^{*}$ abbreviates $\left\{\boldsymbol{X}^{*}: \boldsymbol{X} \in \mathrm{C}\right\}$. (Ostensibly, 34] deals with Heyting algebras, but its argument applies equally to Brouwerian algebras.)

Lemma 10.4. Let $\boldsymbol{Y}, \boldsymbol{Z}, \boldsymbol{W} \in\left\{\boldsymbol{P}_{6}\right\} \cup\left\{\boldsymbol{K}_{n}: n \geq 3\right\}$, where $\boldsymbol{Y} \notin\left\{\boldsymbol{P}_{6}, \boldsymbol{Z}\right\}$. Then $\boldsymbol{Y}^{*} \notin \mathbb{S H}\left(\widehat{\boldsymbol{Z}}^{*}\right)$ and $\boldsymbol{W}^{*} \notin \mathbb{I} \mathbb{S}\left(\widehat{\boldsymbol{Z}}^{*}\right)$.

Proof. Suppose $\boldsymbol{Y}^{*} \in \mathbb{S H}\left(\widehat{\boldsymbol{Z}}^{*}\right)$. Dualizing the injective/surjective homomorphisms, we infer from Lemma 10.2 that there exist $U \in U p(\widehat{\boldsymbol{Z}})$ and a surjective $p$-morphism $g: \boldsymbol{U} \rightarrow \boldsymbol{Y}$. Now $\boldsymbol{Y}$ and $\widehat{\boldsymbol{Z}}$ have depth 3 , and $\boldsymbol{Y}$ has a unique element of depth 3, viz. its least element, $w$ say. As $g$ is surjective, $w=g(u)$ for some $u \in U$. Then $u$ has depth 3, by (8). As $g$ is a $p$-morphism and $w$ has at least three distinct covers, each of depth 2 , the same is true of $u$, by (7) and (8). This prevents $u$ from having the form $e_{x y}$, so $u$ belongs to $\boldsymbol{Z}$. As the least element of $\boldsymbol{Z}$ is its sole element of depth 3, it is $u$. Therefore, $Z \subseteq U$, as $U \in U p(\widehat{\boldsymbol{Z}})$. Moreover, $\uparrow w=\uparrow g(u)=g[\uparrow \widehat{\boldsymbol{Z}} u]$, i.e., $\boldsymbol{Y}=g[\boldsymbol{Z}]$.

Then $\boldsymbol{Z} \neq \boldsymbol{P}_{6}$, because $\boldsymbol{Y}$ has at least eight elements, while $\boldsymbol{P}_{6}$ has only six. Thus, $\boldsymbol{Y}, \boldsymbol{Z}$ are distinct elements of $\left\{\boldsymbol{K}_{n}: n \geq 3\right\}$. As $\left.g\right|_{Z}$ is a surjective $p$-morphism $\boldsymbol{Z} \rightarrow \boldsymbol{Y}$, the homomorphism $\left(\left.g\right|_{Z}\right)^{*}: \boldsymbol{Y}^{*} \rightarrow \boldsymbol{Z}^{*}$ is injective, by Lemma 10.2, so $\boldsymbol{Y}^{*} \in \mathbb{I S}\left(\boldsymbol{Z}^{*}\right)$, whence $\mathbb{V}\left(\boldsymbol{Y}^{*}, \boldsymbol{Z}^{*}\right)=\mathbb{V}\left(\boldsymbol{Z}^{*}\right)$. This contradicts (9), because $\boldsymbol{Y} \neq \boldsymbol{Z}$, so $\boldsymbol{Y}^{*} \notin \mathbb{S H}\left(\widehat{\boldsymbol{Z}}^{*}\right)$.

Now suppose $\boldsymbol{W}^{*} \in \mathbb{I} S\left(\widehat{\boldsymbol{Z}}^{*}\right)$. Then the situation in the first paragraph of the present proof obtains, but with $\boldsymbol{U}=\widehat{\boldsymbol{Z}}$ and $\boldsymbol{Y}=\boldsymbol{W}$. Let $p, q, r$ be 
distinct covers of $w$ in $\boldsymbol{W}$. As we saw above, $u$ has distinct covers $p^{\prime}, q^{\prime}, r^{\prime}$ (of depth 2) that are mapped by $g$ to $p, q, r$, respectively. As $g$ is isotone, $g\left(e_{p^{\prime} q^{\prime}}\right)$ is a common lower bound of the set $\left\{g\left(p^{\prime}\right), g\left(q^{\prime}\right)\right\}=\{p, q\}$, so $g\left(e_{p^{\prime} q^{\prime}}\right)=w$. Then, because $w$ has three distinct covers (of depth 2) in $\boldsymbol{W}$, it follows from (17) and (8) that $e_{p^{\prime} q^{\prime}}$ has three distinct covers (of depth 2) in $\widehat{Z}$, but this contradicts the definitions of $e_{p^{\prime} q^{\prime}}$ and $\widehat{\boldsymbol{Z}}$. Thus, $\boldsymbol{W}^{*} \notin \mathbb{I S}\left(\widehat{\boldsymbol{Z}}^{*}\right)$.

Lemma 10.5. Let $\mathrm{C}=\left\{\widehat{\boldsymbol{P}}_{6}\right\} \cup\{\widehat{\boldsymbol{Z}}: \boldsymbol{Z} \in \mathrm{E}\}$, where $\mathrm{E} \in \mathcal{K}$. Then the variety $\mathbb{V}\left(\mathrm{C}^{*}\right)$ is structurally incomplete.

Proof. Let $\boldsymbol{D}$ be the direct product of the members of $\mathrm{C}^{*}$. Then $\mathbb{V}\left(\mathrm{C}^{*}\right)=$ $\mathbb{V}(\boldsymbol{D})$, so it suffices to show that $\mathbb{V}\left(\mathrm{C}^{*}\right) \neq \mathbb{Q}(\boldsymbol{D})$. As $\boldsymbol{P}_{6}{ }^{*} \in \mathbb{H}\left(\widehat{\boldsymbol{P}}_{6}^{*}\right) \subseteq \mathbb{V}\left(\mathrm{C}^{*}\right)$, it is enough to prove that $\boldsymbol{P}_{6}{ }^{*} \notin \mathbb{Q}(\boldsymbol{D})$. Observe that

$$
\boldsymbol{P}_{6}{ }^{*} \in \mathbb{Q}(\boldsymbol{D}) \text { iff } \boldsymbol{P}_{6}{ }^{*} \in \mathbb{I S P}_{\mathrm{U}}(\boldsymbol{D}) \text { iff } \boldsymbol{P}_{6}{ }^{*} \in \mathbb{I S}(\boldsymbol{D}) \text {. }
$$

The first equivalence obtains because $\mathbb{Q}=\mathbb{P}_{\mathrm{S}} \mathbb{S P}_{\mathrm{U}}$ and $\boldsymbol{P}_{6}{ }^{*}$ is subdirectly irreducible; the second because $\boldsymbol{P}_{6}{ }^{*}$ is finite and of finite type. We must therefore show that $\boldsymbol{P}_{6}{ }^{*} \notin \mathbb{I S}(\boldsymbol{D})$.

Suppose $\boldsymbol{P}_{6}{ }^{*} \in \mathbb{I S}(\boldsymbol{D})$. Then $\boldsymbol{P}_{6}{ }^{*} \in \mathbb{I S P}\left(\mathrm{C}^{*}\right)$. As $\mathbb{S P}=\mathbb{P}_{\mathbb{S}} \mathbb{S}$, it follows (again from the subdirect irreducibility of $\boldsymbol{P}_{6}{ }^{*}$ ) that $\boldsymbol{P}_{6}{ }^{*}$ embeds into $\widehat{\boldsymbol{Z}}^{*}$ for some $\boldsymbol{Z} \in\left\{\boldsymbol{P}_{6}\right\} \cup$ E. This contradicts Lemma 10.4, so $\boldsymbol{P}_{6}{ }^{*} \notin \mathbb{I S}(\boldsymbol{D})$.

Every variety of Dunn monoids (e.g., Brouwerian algebras) has EDPC, by [22, Thm. 3.55]. We shall require the following general result.

Theorem 10.6. ([6], [31, Thm. 6.6]) Let $\mathrm{K}$ be a variety of finite type, with $E D P C$, and let $\boldsymbol{A} \in \mathrm{K}$ be finite and subdirectly irreducible. Then there is a largest subvariety of $\mathrm{K}$ that excludes $\boldsymbol{A}$. It consists of all $\boldsymbol{B} \in \mathrm{K}$ such that $\boldsymbol{A} \notin \mathbb{S H}(\boldsymbol{B})$.

Lemma 10.7. The set $\left\{\mathbb{V}\left(C^{*}\right): C=\left\{\widehat{\boldsymbol{P}}_{6}\right\} \cup\{\widehat{\boldsymbol{Z}}: \boldsymbol{Z} \in \mathrm{E}\}\right.$ for some $\left.\mathrm{E} \in \mathcal{K}\right\}$ is a continuum of varieties of Brouwerian algebras of depth 3 .

Proof. Suppose $\mathrm{E} \cup\{\boldsymbol{Y}\} \in \mathcal{K}$, where $\boldsymbol{Y} \notin\left\{\boldsymbol{P}_{6}\right\} \cup$ E. It suffices to show that $\widehat{\boldsymbol{Y}}^{*} \notin \mathbb{V}\left(\left\{\widehat{\boldsymbol{P}}_{6}^{*}\right\} \cup\left\{\widehat{\boldsymbol{Z}}^{*}: \boldsymbol{Z} \in \mathrm{E}\right\}\right)$. As $\boldsymbol{Y}^{*} \in \mathbb{H}\left(\widehat{\boldsymbol{Y}}^{*}\right)$, it is enough to prove that $\boldsymbol{Y}^{*} \notin \mathbb{V}\left(\left\{\widehat{\boldsymbol{P}}_{6}^{*}\right\} \cup\left\{\widehat{\boldsymbol{Z}}^{*}: \boldsymbol{Z} \in \mathrm{E}\right\}\right)$.

Let $\mathrm{G}$ be the class of all Brouwerian algebras $\boldsymbol{B}$ such that $\boldsymbol{Y}^{*} \notin \mathbb{S H}(\boldsymbol{B})$. Since $\boldsymbol{Y}^{*}$ is finite and subdirectly irreducible, Theorem 10.6 shows that, for any variety $\mathrm{K}$ of Brouwerian algebras, we have $\boldsymbol{Y}^{*} \notin \mathrm{K}$ iff $\mathrm{K} \subseteq \mathrm{G}$. Therefore, it remains only to confirm that $\left\{\widehat{\boldsymbol{P}}_{6}^{*}\right\} \cup\left\{\widehat{\boldsymbol{Z}}^{*}: \boldsymbol{Z} \in \mathrm{E}\right\} \subseteq \mathrm{G}$, i.e., that $\boldsymbol{Y}^{*} \notin \mathbb{S H}\left(\widehat{\boldsymbol{P}}_{6}^{*}\right)$ and $\boldsymbol{Y}^{*} \notin \mathbb{S H}\left(\widehat{\boldsymbol{Z}}^{*}\right)$ for all $\boldsymbol{Z} \in \mathrm{E}$. This is indeed the case, by Lemma 10.4, because $\boldsymbol{Y} \notin\left\{\boldsymbol{P}_{6}\right\} \cup$ E.

Theorem 10.8. The variety of Brouwerian algebras has $2^{\aleph_{0}}$ structurally incomplete subvarieties (of depth 3).

Proof. Use Lemmas 10.5 and 10.7. 
This also completes the proof of Theorem 9.6. When we switch from Brouwerian to Heyting algebras, the duality theory undergoes slight definitional changes (see [5, Sec. 4], for instance). The proof of Theorem 10.8 adapts easily, however, and the result remains true when we replace 'Brouwerian' by 'Heyting' in its statement.

In a quasivariety $\mathrm{K}$, a quasi-equation

$$
\left(\varphi_{1} \approx \psi_{1} \& \cdots \& \varphi_{n} \approx \psi_{n}\right) \Longrightarrow \varphi \approx \psi
$$

is said to be admissible provided that, for every substitution $h$,

$$
\text { if } \mathrm{K} \models h\left(\varphi_{i}\right) \approx h\left(\psi_{i}\right) \text { for } i=1, \ldots, n \text {, then } \mathrm{K} \models h(\varphi) \approx h(\psi) \text {. }
$$

By Theorem 6.1, $\mathrm{K}$ is $\mathrm{SC}$ iff it satisfies its own admissible quasi-equations.

Mints [47] showed (in effect) that the variety BRA of all Brouwerian algebras is not $\mathrm{SC}$, by proving that the following quasi-equation (not satisfied by BRA) is admissible in BRA:

$$
x \rightarrow y \leqslant x \vee z \Longrightarrow((x \rightarrow y) \rightarrow x) \vee((x \rightarrow y) \rightarrow z) \approx e .
$$

For each term $\varphi$ over BRA, we define a term $\varphi^{\diamond}$ in the following recursive manner: $e^{\diamond}=e ; x^{\diamond}=x \wedge e(x \in \operatorname{Var}) ;(\alpha * \beta)^{\diamond}=\alpha^{\diamond} * \beta^{\diamond}(* \in\{\wedge, \vee\})$; $(\alpha \rightarrow \beta)^{\diamond}=\left(\alpha^{\diamond} \rightarrow \beta^{\diamond}\right) \wedge e$. In this signature, the amendment $\Phi^{\diamond}$ of a quasi-equation $\Phi$ results from replacing each term $\varphi$ occurring in $\Phi$ with the term $\varphi^{\diamond}$. Using ideas of Iemhoff [27, one can prove that if a quasi-equation $\Phi$ is admissible in BRA, then $\Phi^{\diamond}$ is admissible in the variety DM of all Dunn monoids; moreover, if BRA $\forall \varnothing$, then DM $\forall \models \Phi^{\diamond}$. This implies, of course, that DM is not SC.

It is tempting to try to extend the argument from DM to the variety $\mathrm{M}$ of Definition 8.5, using reflections. Unfortunately, however, it turns out that the amendment of (10) is not admissible in $\mathrm{M}$.

Acknowledgment. The first author thanks Miguel Campercholi for stimulating conversations on this topic.

\section{REFERENCES}

[1] A.R. Anderson, N.D. Belnap, Jnr., 'Entailment: The Logic of Relevance and Necessity, Vol. 1', Princeton University Press, 1975.

[2] A. Avron, What is relevance logic?, Ann. Pure Appl. Logic 165 (2014), 26-48.

[3] C. Bergman, Structural completeness in algebra and logic, in H. Andréka, J.D. Monk and I. Nemeti (eds.), 'Algebraic Logic', Colloquia Mathematica Societatis János Bolyai Vol. 54, North-Holland, Amsterdam, 1991, pp. 59-73.

[4] C. Bergman, R. McKenzie, Minimal varieties and quasivarieties, J. Austral. Math. Soc. Ser. A 48 (1990), 133-147.

[5] G. Bezhanishvili, T. Moraschini, J.G. Raftery, Epimorphisms in varieties of residuated structures, J. Algebra 492 (2017), 185-211.

[6] W.J. Blok, D. Pigozzi, On the structure of varieties with equationally definable principal congruences I, Algebra Universalis 15 (1982), 195-227.

[7] W.J. Blok, D. Pigozzi, 'Algebraizable Logics', Memoirs of the American Mathematical Society 396, Amer. Math. Soc., Providence, 1989. 
[8] S. Burris, H.P. Sankappanavar, 'A Course in Universal Algebra', Graduate Texts in Mathematics, Springer-Verlag, New York, 1981.

[9] M.A. Campercholi, D.J. Vaggione, Implicit definition of the quaternary discriminator, Algebra Universalis 68 (2012), 1-16.

[10] C.C. Chang, H.J. Keisler, 'Model Theory', 3rd ed., Elsevier, Amsterdam, 1990.

[11] P. Cintula, G. Metcalfe, Structural completeness in fuzzy logics, Notre Dame J. Form. Log. 50 (2009), 153-182.

[12] P. Cintula, G. Metcalfe, Admissible rules in the implication-negation fragment of intuitionistic logic, Ann. Pure Appl. Logic 162 (2010), 162-171.

[13] A.I. Citkin, On structurally complete superintuitionistic logics, Soviet Math. Dokl. 19 (1978), 816-819.

[14] A.I. Citkin, Hereditarily structurally complete positive logics. Manuscript.

[15] J.M. Dunn, 'The Algebra of Intensional Logics', PhD thesis, University of Pittsburgh, 1966.

[16] Z. Dywan, Decidability of structural completeness for strongly finite propositional calculi, Bull. Sect. Logic 7 (1978), 129-132.

[17] W. Dzik, M.M. Stronkowski, Almost structural completeness; an algebraic approach, Ann. Pure Appl. Logic 167 (2016), 525-556.

[18] W. Dziobiak, There are $2^{\aleph_{0}}$ logics with the relevance principle between $R$ and $R M$, Studia Logica 42 (1983), 49-61.

[19] L.L. Esakia, Topological Kripke models, Soviet Math. Dokl. 15 (1974), 147-151.

[20] L.L. Esakia, 'Heyting Algebras I. Duality Theory', Metsniereba Press, Tblisi, 1985 (Russian).

[21] J.M. Font, G. Rodríguez, Note on algebraic models for relevance logic, Zeitschr. f. math. Logik und Grundlagen der Math. 36 (1990), 535-540.

[22] N. Galatos, P. Jipsen, T. Kowalski, H. Ono, 'Residuated Lattices. An Algebraic Glimpse at Substructural Logics', Studies in Logic and the Foundations of Mathematics 151, Elsevier, 2007.

[23] N. Galatos, J.G. Raftery, A category equivalence for odd Sugihara monoids and its applications, J. Pure Appl. Algebra 216 (2012), 2177-2192.

[24] V.A. Gorbunov, The cardinality of subdirectly irreducible systems in quasivarieties, Algebra and Logic 25 (1986), 1-34.

[25] V.A. Gorbunov, 'Algebraic Theory of Quasivarieties', Consultants Bureau, New York, 1998.

[26] A. Hsieh, J.G. Raftery, Conserving involution in residuated structures, MLQ Math. Log. Q. 53 (2007), 583-609.

[27] R. Iemhoff, On the admissible rules of intuitionistic propositional logic, J. Symb. Log. 66 (2001), 281-294.

[28] A. Ignjatović, M.Z. Grulović, A comment on the joint embedding property, Period. Math. Hungar. 33 (1996), 45-50.

[29] B. Jónsson, Algebras whose congruence lattices are distributive, Math. Scand. 21 (1967), 110-121.

[30] B. Jońsson, 'Topics in universal algebra', Lecture Notes in Math., Vol. 250, SpringerVerlag, Berlin and New York, 1972.

[31] B. Jónsson, Congruence distributive varieties, Math. Japonica 42 (1995), 353-401.

[32] H. Kihara, H. Ono, Interpolation properties, Beth definability properties and amalgamation properties for substructural logics, J. Logic Comput. 20 (2010), 823-875.

[33] J. Kollár, Congruences and one-element subalgebras, Algebra Universalis 9 (1979), 266-267.

[34] A.V. Kuznetsov, On superintuitionistic logics, Proceedings of the International Congress of Mathematicians, Vancouver, BC, 1974, Vol. 1, Canadian Mathematical Congress, Montreal, Quebec, 1975, pp. 243-249. 
[35] J. Łoś, Common extension in equational classes, in E. Nagel, P. Suppes, A. Tarksi, 'Logic, Methodology and Philosophy of Science. Proceedings of the 1960 International Conference', Stanford University Press, Stanford, 1962, pp. 136-142.

[36] J. Łoś, R. Suszko, Remarks on sentential logics, Proc. Kon. Nederl. Akad. van Wetenschappen, Series A 61 (1958), 177-183.

[37] R. Magari, Una dimonstrazione del fatto che ogni varietà ammette algebre semplici, Ann. Univ. Ferrara, Sez. VII 14 (1969), 1-4.

[38] L.L. Maksimova, The principle of separation of variables in propositional logics, Algebra and Logic 15 (1976), 105-114.

[39] L.L. Maksimova, On variable separation in modal and superintuitionistic logics, Studia Logica 55 (1995), 99-112.

[40] A.I. Maltsev, Several remarks on quasivarieties of algebraic systems, Algebra i Logika 5 (1966), 3-9 (Russian). English translation in 41, pp. 416-421].

[41] A.I. Maltsev, 'The Metamathematics of Algebraic Systems. Collected Papers: 19361967' (B.F. Wells, III, ed.), North-Holland, Amsterdam, 1971.

[42] A.I. Maltsev, 'Algebraic Systems', Springer-Verlag, Berlin, 1973.

[43] G. Metcalfe, C. Röthlisberger, Admissibility in finitely generated quasivarieties, Log. Methods Comput. Sci. 9 (2013), 1-19.

[44] R.K. Meyer, On conserving positive logics, Notre Dame J. Form. Log. 14 (1973), $224-236$.

[45] R.K. Meyer, J.M. Dunn, H. Leblanc, Completeness of relevant quantification theories, Notre Dame J. Form. Log. 15 (1974), 97-121.

[46] P. Minari, A. Wroński, The property (HD) in intermediate logics. A partial solution of a problem of H. Ono, Rep. Math. Logic 22 (1988), 21-25.

[47] G.E. Mints, Derivability of admissible rules, J. Soviet Math. 6 (1976), 417-421.

[48] T. Moraschini, J.G. Raftery, J.J. Wannenburg, Varieties of De Morgan monoids: minimality and irreducible algebras, J. Pure Appl. Algebra, in press: https://doi.org/10.1016/j.jpaa.2018.09.015

[49] T. Moraschini, J.G. Raftery, J.J. Wannenburg, Varieties of De Morgan monoids: covers of atoms, Rev. Symb. Log., in press: https://doi.org/10.1017/S1755020318000448

[50] J.S. Olson, J.G. Raftery, Positive Sugihara monoids, Algebra Universalis 57 (2007), 75-99.

[51] J.S. Olson, J.G. Raftery, C.J. van Alten, Structural completeness in substructural logics, Log. J. IGPL 16 (2008), 453-495.

[52] W.A. Pogorzelski, Structural completeness of the propositional calculus, Bull. de l'Acad. Polonaise des Sciences 19 (1971), 345-351.

[53] W.A. Pogorzelski, P. Wojtylak, 'Completeness Theory for Propositional Logics', Studies in Universal Logic, Birkhäuser, Basel-Boston-Berlin, 2008.

[54] B. Poizat, A. Yeshkeyev, Positive Jonsson theories, Log. Univers. 12 (2018), 101-127.

[55] T. Prucnal, On the structural completeness of some pure implicational propositional calculi, Studia Logica 30 (1972), 45-52.

[56] T. Prucnal, Structural completeness of some fragments of intermediate logics, Bull. Sect. Logic 12 (1983), 41-44.

[57] J.G. Raftery, Admissible rules and the Leibniz hierarchy, Notre Dame J. Form. Log. 57 (2016), 569-606.

[58] J.G. Raftery, K. Świrydowicz, Structural completeness in relevance logics, Studia Logica 104 (2016), 381-387.

[59] V.V. Rybakov, Hereditarily structurally complete modal logics, J. Symb. Log. 60 (1995), 266-288.

[60] V.V. Rybakov, 'Admissibility of Logical Inference Rules', Studies in Logic and the Foundations of Mathematics 136, Elsevier, Amsterdam, 1997. 
[61] J.K. Slaney, 3088 varieties: a solution to the Ackermann constant problem, J. Symb. Log. 50 (1985), 487-501.

[62] J.K. Slaney, On the structure of De Morgan monoids with corollaries on relevant logic and theories, Notre Dame J. Form. Log. 30 (1989), 117-129.

[63] J.K. Slaney, R.K. Meyer, A structurally complete fragment of relevant logic, Notre Dame J. Form. Log. 33 (1992), 561-566.

[64] M.M. Stronkowski, Deciding active structural completeness. Manuscript.

[65] M. Tokarz, The existence of matrices strongly adequate for $E, R$ and their fragments, Studia Logica 38 (1979), 75-85.

[66] A. Wroński, On cardinalities of matrices strongly adequate for the intuitionistic propositional logic, Rep. Math. Logic 2 (1974), 55-62.

[67] A. Wroński, On factoring by compact congruences in algebras of certain varieties related to the intuitionistic logic, Bull. Sect. Logic 15 (1986) 48-51.

[68] A. Wroński, Overflow rules and a weakening of structural completeness, in J. SytnikCzetwertyński (ed.), 'Rozważania o Filozofii Prawdziwej. Jerzemu Perzanowskiemu w Darze', Wydawnictwo Uniwersytetu Jagiellońskiego, Kraków, 2009, pp. 67-71.

[69] H. Zeitler, 'Modeltheoretic Investigations on the Amalgamation Property', Ph.D. Thesis, Heidelberg, 1976.

Institute of Computer Science, Academy of Sciences of the Czech Republic, Pod Vodárenskou věží 2, 18207 Prague 8, Czech Republic.

E-mail address: moraschini@cs.cas.cz

Department of Mathematics and Applied Mathematics, University of Pretoria, Private Bag X20, Hatfield, Pretoria 0028, and DST-NRF Centre of ExCellence in Mathematical and Statistical Sciences (CoE-MaSS), South Africa

E-mail address: james.raftery@up.ac.za

Department of Mathematics and Applied Mathematics, University of Pretoria, Private Bag X20, Hatfield, Pretoria 0028, and DST-NRF Centre of ExCellence in Mathematical and Statistical Sciences (CoE-MaSS), South Africa

E-mail address: jamie.wannenburg@up.ac.za 